PeCelveo

FFo $\cap$ b iss

\title{
OSTI
}

\section{Measurement of Charge Exchange Cross Sections for Highly Charged Xenon and Thorium Ions with Molecular Hydrogen in a Penning Ion Trap}

George Morton Weinberg

Ph.D Thesis

December 1995

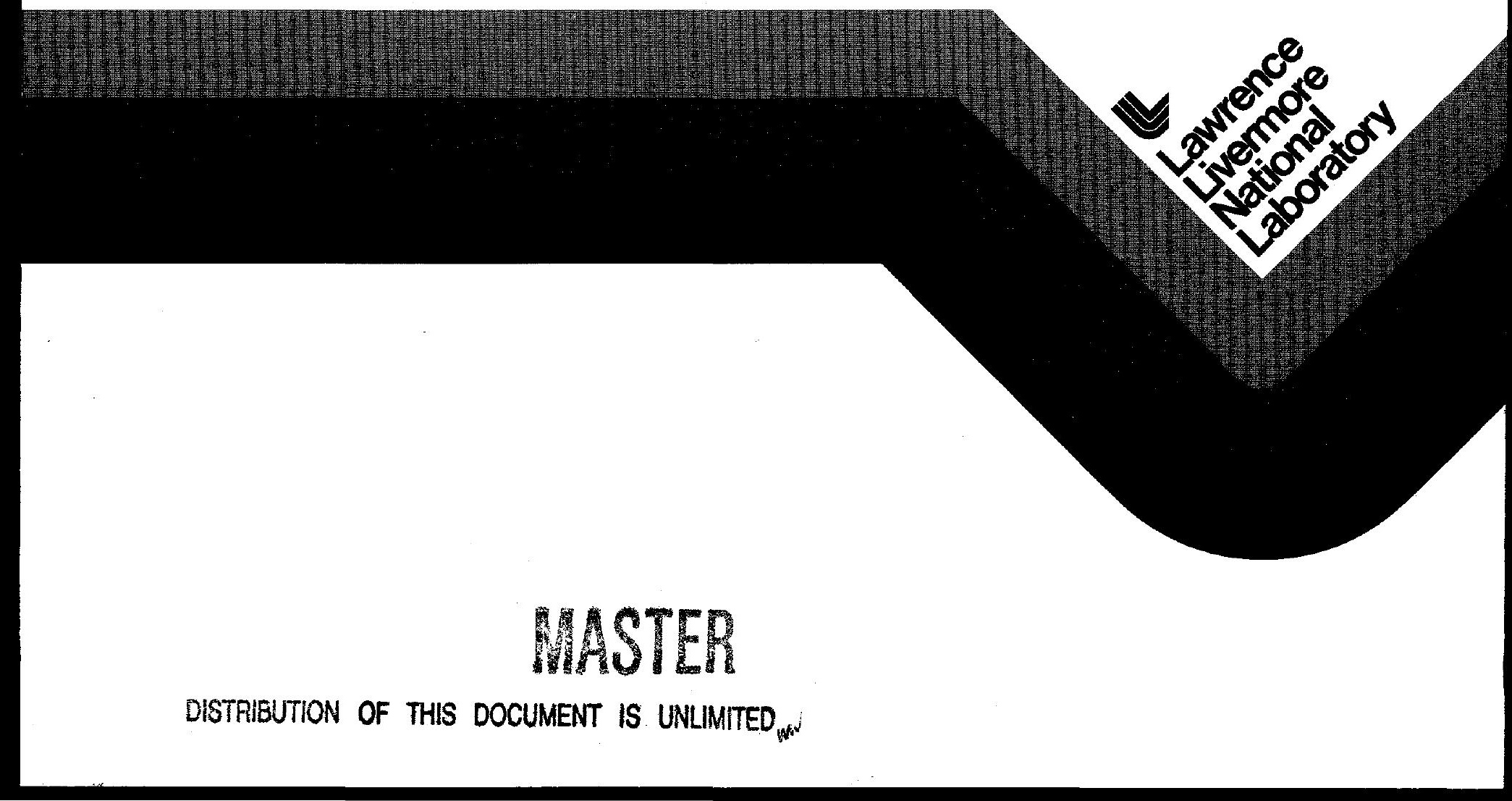




\section{DISCLAIMER}

This document was prepared as an account of work sponsored by an agency of the United States Government. Neither the United States Government nor the University of California nor any of their employees, makes any warranty, express or implied, or assumes any legal liability or responsibility for the accuracy, completeness, or usefulness of any information, apparatus, product, or process disclosed, or represents that its use would not infringe privately owned rights. Reference herein to any specific commercial product, process, or service by trade name, trademark, manufacturer, or otherwise, does not necessarily constitute or imply its endorsement, recommendation, or favoring by the United States Government or the University of California. The views and opinions of authors expressed herein do not necessarily state or reflect those of the United States Government or the University of California, and shall not be used for advertising or product endorsement purposes.

This report has been reproduced directly from the best available copy.

Available to DOE and DOE contractors from the Office of Scientific and Technical Information P.O. Box 62, Oak Ridge, TN 37831

Prices available from (615) 576-8401, FTS 626-8401

Available to the public from the National Technical Information Service

U.S. Department of Commerce

5285 Port Royal Rd.,

Springfield, VA 22161

Work performed under the auspices of the U.S. Department of Energy by Lawrence Livermore National Laboratory under Contract W-7405-ENG-48. 
UCRL-LR-122695

Distribution Category UC-700

\section{Measurement of Charge Exchange Cross Sections for Highly Charged Xenon and Thorium Ions with Molecular Hydrogen in a Penning Ion Trap}

George Morton Weinberg

Manuscript date: December 1995

LAWRENCE LIVERMORE NATIONAL LABORATORY

University of California - Livermore, California 94551 

MEASUREMENT OF CHARGE EXCHANGE CROSS SECTIONS FOR HIGHLY CHARGED XENON AND THORIUM IONS WITH MOLECULAR HYDROGEN IN A PENNING ION TRAP

\author{
A Dissertation \\ by \\ GEORGE MORTON WEINBERG
}

Submitted to the Office of Graduate Studies of

Texas A\&M University

in partial fulfillment of the requirements for the degree of

DOCTOR OF PHILOSOPHY

December 1995

Major Subject: Physics 


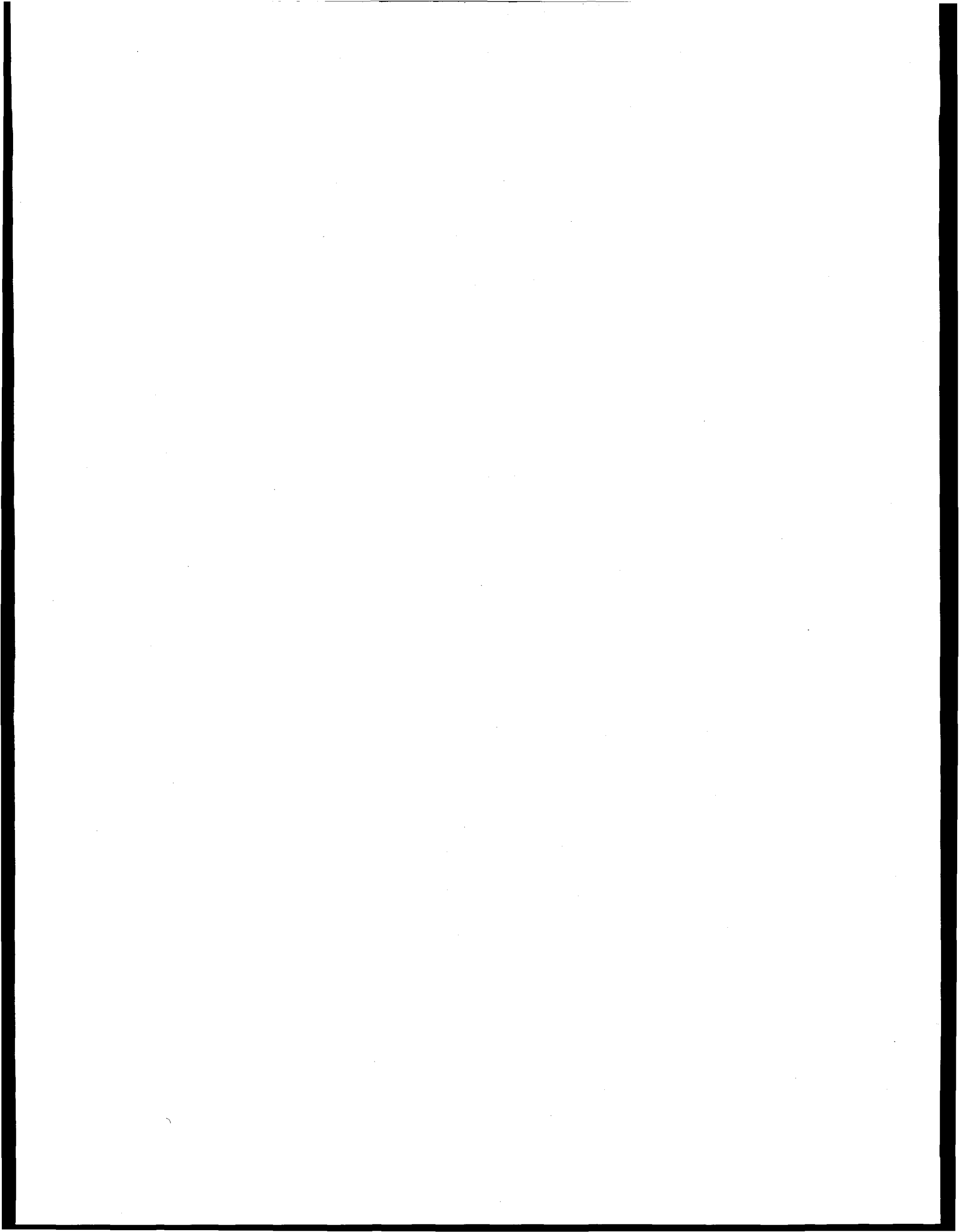




\title{
MEASUREMENT OF CHARGE EXCHANGE CROSS SECTIONS FOR HIGHLY CHARGED XENON AND THORIUM IONS WITH MOLECULAR HYDROGEN IN A PENNING ION TRAP
}

\author{
A Dissertation \\ by \\ GEORGE MORTON WEINBERG \\ Submitted to Texas A\&M University \\ in partial fulfillment of the requirements \\ for the degree of \\ DOCTOR OF PHILOSOPHY
}

Approved as to style and content by:

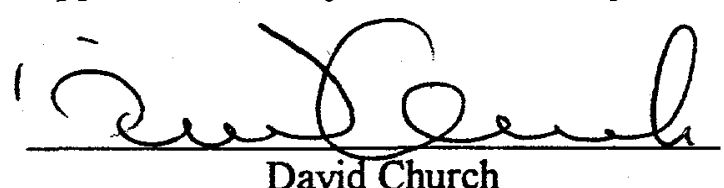

David Church

(Chair of Committee)

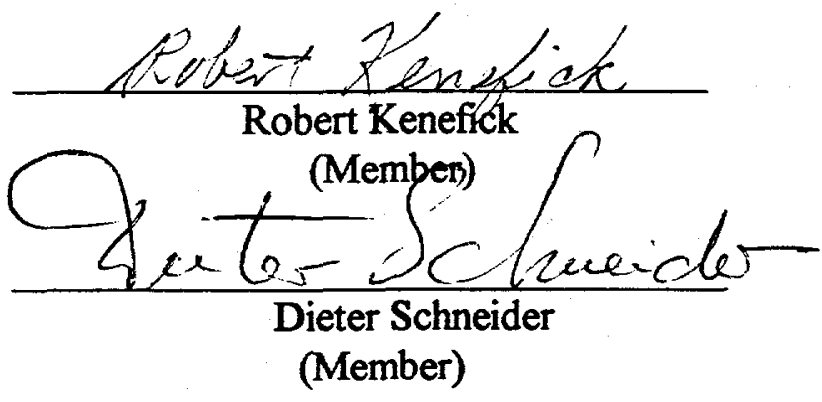

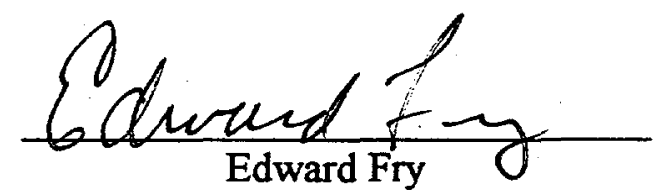

(Member)

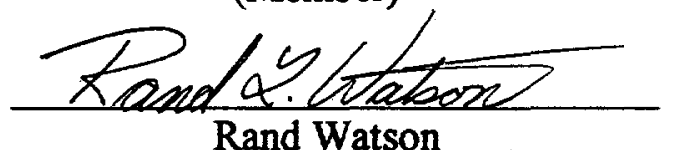

Rand Watson

(Member)

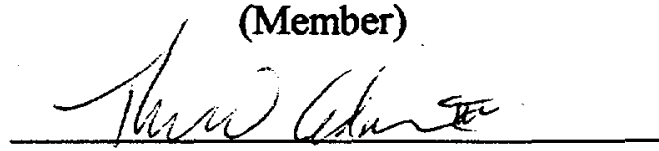

Thomas W. Adair, III

(Head of Department)

December 1995

Major Subject: Physics 



\begin{abstract}
Measurement of Charge Exchange Cross Sections for Highly Charged Xenon and Thorium Ions with Molecular Hydrogen in a Penning Ion Trap. (December 1995) George Morton Weinberg, B.A., University of Illinois Chair of Advisory Committee: Dr. David Church
\end{abstract}

Highly charged xenon ( $35+$ to $46+)$ and thorium ( $72+$ to $79+$ ) ions were produced in an Electron Beam Ion Trap (EBIT). The ions were extracted from EBIT in a short pulse. Ions of one charge state were selected using an electromagnet. The ions were recaptured at low energy in a cryogenic Penning trap (RETRAP). As the ions captured electrons from molecular hydrogen, populations of the various charge states were obtained by measuring the image currents induced by the ions on the electrodes of the trap. Data on the number of ions in each charge state vs. time were compared to theoretical rate equations in order to determine the average charge exchange rates. These rates were compared to charge exchange rates of an ion with a known charge exchange cross section $\left(\mathrm{Ar}^{11+}\right)$ measured in a similar manner in order to determine the average charge exchange cross sections for the highly charged ions. The energy of interaction between the highly charged ions and hydrogen was estimated to be $4 \mathrm{eV}$ in the center of mass frame.

The mean charge exchange cross sections were $9 \times 10^{-14} \mathrm{~cm}^{2}$ for $\mathrm{Xe}^{43+}$ to $\mathrm{Xe}^{46+}$ and $2 \times 10^{-13} \mathrm{~cm}^{2}$ for $\mathrm{Th}^{73+}$ to $\mathrm{Th}^{79+}$. Double capture was approximately $20-25 \%$ of the total for both xenon and thorium. A fit indicated that the cross sections were approximately proportional to $q^{1.2}$. This is consistent with a linear dependence of cross section on $\mathrm{q}$ within the measurement uncertainties. 


\section{ACKNOWLEDGMENTS}

I would like to thank my committee chairman, David Church, for his support and guidance, and Dieter Schneider for his capable leadership of the EBIT-RETRAP project.

I would also like to thank the other memebers of the RETRAP team (Joachim Steiger, Bret Beck, Lukas Gruber, and Christiana Ruehliche). Working with them has always been educational, often in areas almost completely unrelated to atomic physics.

And a special thanks to Joe McDonald, who in operating EBIT has shown perserverence worthy of the legendary postal workers of old.

Finally I would like to thank Dan Nelson and Ed Magee for providing technical support.

This work was performed under the auspices of the U. S. Department of Energy by Lawrence Livermore National Laboratory under contract No. W-7405-ENG-48. 
Page

ABSTRACT

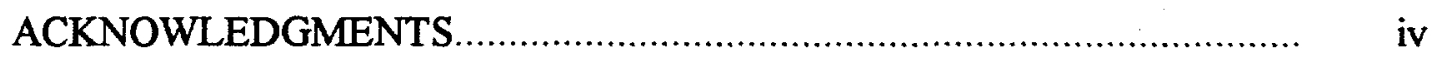

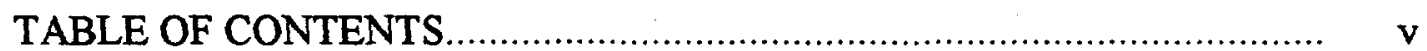

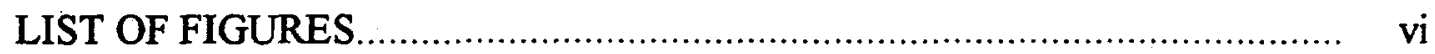

CHAPTER

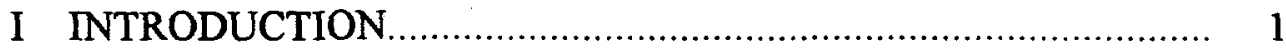

II BACKGROUND

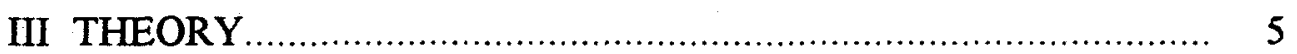

IV EXPERIMENTAL METHOD................................................ 12

A. The Electron Beam Ion Trap (EBIT) ………….............. 12

B. Ion Extraction and Transport........................................ 12

C. Ion Capture ……......................................................... 18

D. The Penning Trap.......................................................... 23

E. Measurement of Ion Signals............................................. 29

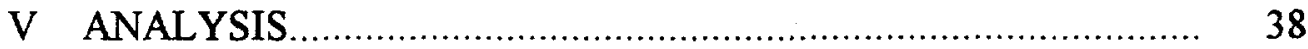

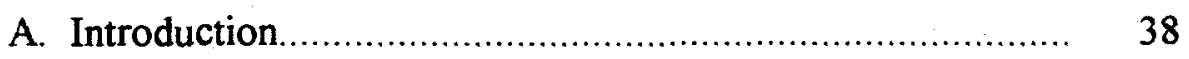

B. Digital Mode ........................................................... 38

C. Analog Mode

D. Rate Equations .......................................................... 47

E. Neutral Density Calibration............................................... 51

F. Results and Discussion.................................................. 57

G. Comparison with Theory ................................................. 60

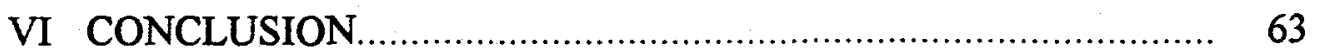

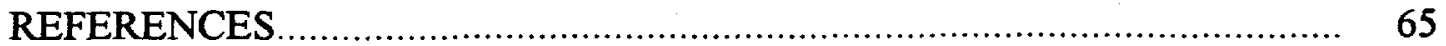

VITA 


\section{LIST OF FIGURES}

Figure 3.1: Theoretical Electron Capture Cross-Section vs. Energy for Heavy Ions and Molecular Hydrogen.

Figure 3.2: Theoretical Electron Capture Cross-Section vs. Charge for Heavy Ions and Molecular Hydrogen................................ 7

Figure 4.1: EBIT-RETRAP System Schematic .............................. 15

Figure 4.2: Deceleration Tube Circuit Schematic.......................... 19

Figure 4.3: Endcap Electrode Circuit Schematic............................ 22

Figure 4.4: The Penning Trap............................................... 24

Figure 4.5: Potential Along the Trap Axis.................................... 26

Figure 4.6: Frequency Dependence on Ion Amplitude and Radius ......... 27

Figure 4.7: Injected Ion Signal Average Without Predump................. 31

Figure 4.8: Injected Ion Signal Average With Predump....................... 32

Figure 4.9: Endcap Electrode Voltages During Predump-Precool........... 34

Figure 4.10: Ion Signal Average After 80 Seconds Storage Time............ 36

Figure 4.11: Trapping Cycle Schematic.................................... 37

Figure 5.1: Individual Ion Signals.............................................. 39

Figure 5.2: Analog Mode Ion Signal Data.................................... 43

Figure 5.3: Corrected Analog Mode Ion Signal Data....................... 48

Figure 5.4: $\mathrm{Be}^{2+}$ Signal vs. Time ............................................. 54

Figure 5.5: $\mathrm{Be}^{1+}$ Signal vs. Time................................................. 55

Figure 5.6: Total Charge Exchange Cross-Section Data Summary.......... 59 


\section{CHAPTER I}

\section{INTRODUCTION}

This dissertation describes experiments investigating basic atomic collision processes by studying charge transfer reactions between very highly charged ions $(35+$ to $79+$ ) and molecular hydrogen at very low energies (about $4 \mathrm{eV}$ in the center of mass frame). The highly charged ions were produced in an electon beam ion trap (EBIT), extracted in a short pulse ( $\sim 10$ microseconds) and one charge state $q$ was selected. The ions were then compressed and recaptured in a cryogenic Penning trap (RETRAP). As the ions capture electrons from the background hydrogen, the populations of various charge states at regular intervals were calculated from nondestructive measurements of image currents that the ions induced on the electrodes of the trap.

By obtaining the number of ions in various charge states versus time, the charge exchange rates $k$ were calculated. These in turn yield the mean charge exchange cross sections via the equation

$\mathrm{k}_{\mathrm{q}}=\mathrm{n} \int_{0}^{\infty} \sigma_{q}[s] f[s] s d s=\sigma_{q} \bar{s} \mathrm{n}$

where $\mathrm{f}[\mathrm{s}]$ is the distribution of ions of speed $\mathrm{s}$ in the trap, $\sigma_{\mathrm{q}}[\mathrm{s}]$ is the charge exchange cross section in $\mathrm{cm}^{2}$ for ions of charge $\mathrm{q}$ as a function of speed, $\sigma_{\mathrm{q}}$ is the mean charge exchange cross section, $\mathrm{s}$ is the ion speed in $\mathrm{cm} / \mathrm{sec}, \bar{s}$ is the average speed and $\mathrm{n}$ is the target density in $\mathrm{cm}^{-3}$. The target density is calculated by measuring reaction rates of low charge state ions with known charge exchange cross sections.

As preparation for these measurements the RETRAP system was designed,

Citations in this dissertation follow the format of Physical Review A. 
assembled, and tested as part of a collaboration. Aspects of this system, to which major contributions were made by the author, are discussed in some detail. 


\section{CHAPTER II}

\section{BACKGROUND}

Many previous experiments have made use of ion traps to study the properties of ions at low energies [1], including charge transfer cross sections. However, the ions involved are generally of fairly low charge (the highest charge state mentioned in the above reference is (in units of the fundamental charge) 10+).

Charge transfer measurements have also been made using ion beams. These experiments are usually made at relatively high energy (10keV / a.m.u is considered "low energy"), although a few measurements have been made at less than $1 \mathrm{eV}$ [2],[3].

These, however, used ions of charge $11+$ or less.

Ion storage rings [4] are capable of producing very highly charged ions for a variety of experiments including interactions with neutrals[5]. However, storage ring experiments are done at a much higher energy ( $10 \mathrm{MeV} / \mathrm{a} . \mathrm{m} . \mathrm{u}$.$) than the experiments$ described herein.

Highly charged ions are also produced by sputtering from the walls of tokamaks. These will generally be iron or lighter elements, although heavier elements may exist as impurities, and of course they can be deliberately introduced into tokamaks for experimental purposes. Beams of neutrals are injected into tokamaks and the interactions between the ions and the neutrals are studied for diagnostic purposes[6]. Again, however, energies are much higher than in RETRAP (typically about 25 keV/a.m.u.).

Ions with charge of up to about 45 can be produced by an electron beam ion source (EBIS), and ions of even higher charge can be produced in an electron beam ion trap (EBIT). Conceptually, an EBIS and an EBIT are very similar. Both ionize atoms via electron beam impact and store them in an electrostatic potential well. The region in which ions are stored in an EBIT is shorter because it was originally designed as an Xray source, and it is more important to have a small ion volume than a large ion number. 
Plasma instabilities which increase with length limit the maximum charge state of an EBIS [7], so an EBIT can achieve a higher maximum charge. Extraction of ions from an EBIT has been routinely performed for several years.

Inside an EBIT there is a distribution of charge states. Ions extracted from EBIT can be charge state analyzed, but extraction at potentials below about $1 \mathrm{keV}$ are very difficult, and even a very slow ion in a beam is present in a given volume for a very short time (for example, a xenon $44+$ ion accelerated by a potential of 1 volt will pass through an experimental chamber $10 \mathrm{~cm}$ long in $13 \mathrm{usec}$ ).

In RETRAP it is possible to study a single charge state of very highly charged ions (Th $80+$ has been captured) at energies of only a few volts times the charge (and even lower energies after cooling; see Conclusion). Low charge state light ions (such as $\mathrm{Be}^{2+}$ ) remain in RETRAP for hours, although the lifetimes of the individual charge states of highly charged ions are limited by charge exchange rates. RETRAP thus allows experiments in a virtually unexplored region of energies for very highly charged ions.

It was inevitable that charge exchange reaction rates with molecular hydrogen would be among the first experiments performed at RETRAP, since hydrogen is the dominant background gas in cryogenically pumped systems. Ion storage times are necessary information for planning and interpreting virtually all other RETRAP experiments. Use in future RETRAP experiments may be regarded as the primary motivation for these charge exchange measurements. They also provide a test of basic theories of charge transfer processes.

Xenon $44+$ and $45+$ were chosen for study because they are reasonably high charge state ions which EBIT can easily produce in large numbers, and because of the large difference in potential energy compared to the small change in charge (ionizing Xe $43+$ to $44+$ requires $3.3 \mathrm{keV}$; ionizing $44+$ to $45+$ requires $7.7 \mathrm{keV}$ ). Thorium was chosen to extend these measurements to the highest charge states achievable. 


\section{CHAPTER III}

\section{THEORY}

Before considering the various theories of electron capture by highly charged ions at low energies, certain common features of the theories should be pointed out. First, it is expected that electrons from neutrals will usually be captured into high energy levels of highly charged ions, so the nucleus and remaining electrons of the highly charged ion can be treated as a point charge before charge exchange. Second, charge exchange processes are fast relative to the time the ion and atom spend near each other, so cross section dependence on energy is expected to be weak. The Langevin theory is an exception to this second rule, as will be seen below.

Atomic units are used throughout this section except where otherwise stated, and the initial charge on the ion is designated $z$. Figure 3.1 shows the dependence of each theory on energy for a constant charge (76+). Figure 3.2 shows the dependence on charge for a constant energy ( $2 \mathrm{eV})$. Let us first consider Grozdanov's classical model of charge exchange [8]. This model was developed for charge transfer between hydrogenlike ions and hydrogen. The electron is considered to move in a field of two centers (the nuclei of the ion and the neutral). The Hamiltonian function of the electron is given by

$\mathrm{H}=\mathrm{p}^{2} / 2-1 / \mathrm{r}_{1}-\mathrm{z} / \mathrm{r}_{2}$

where $r_{1}$ is the distance to the proton and $r_{2}$ is the distance to the ion nucleus. A change of variables was made,

$\xi=\left(r_{1}+r_{2}\right) / R$

$\eta=\left(\mathbf{r}_{1}-\mathbf{r}_{2}\right) / \mathbf{R}$ 


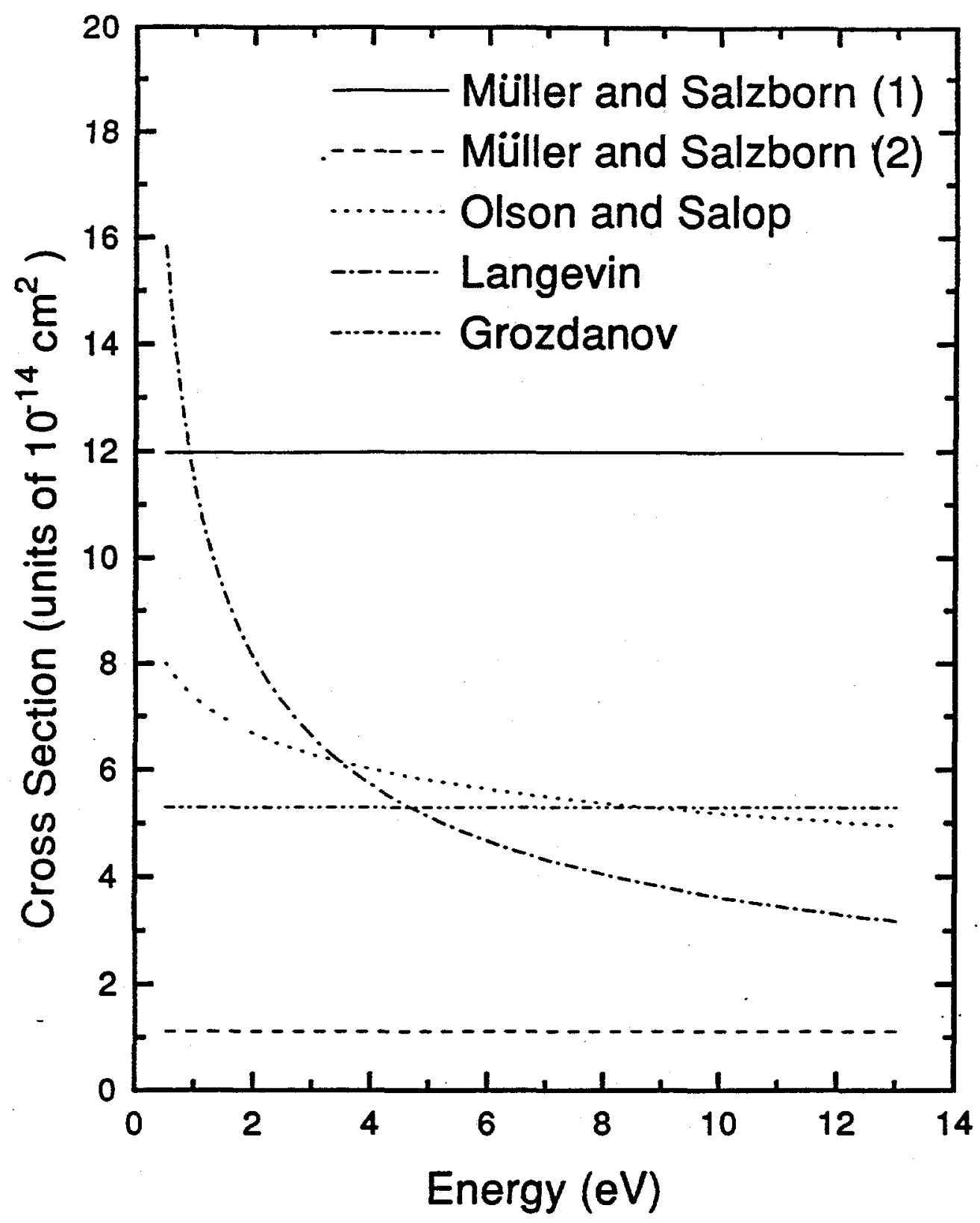

Figure 3.1: Theoretical electron capture cross-section vs. energy for heavy ions and molecular hydrogen. The charge is held constant $(q=76)$. Energy given is in the center of mass frame. The first Muller and Salzbom curve is for single electron capture, the second is for double electron capture. 


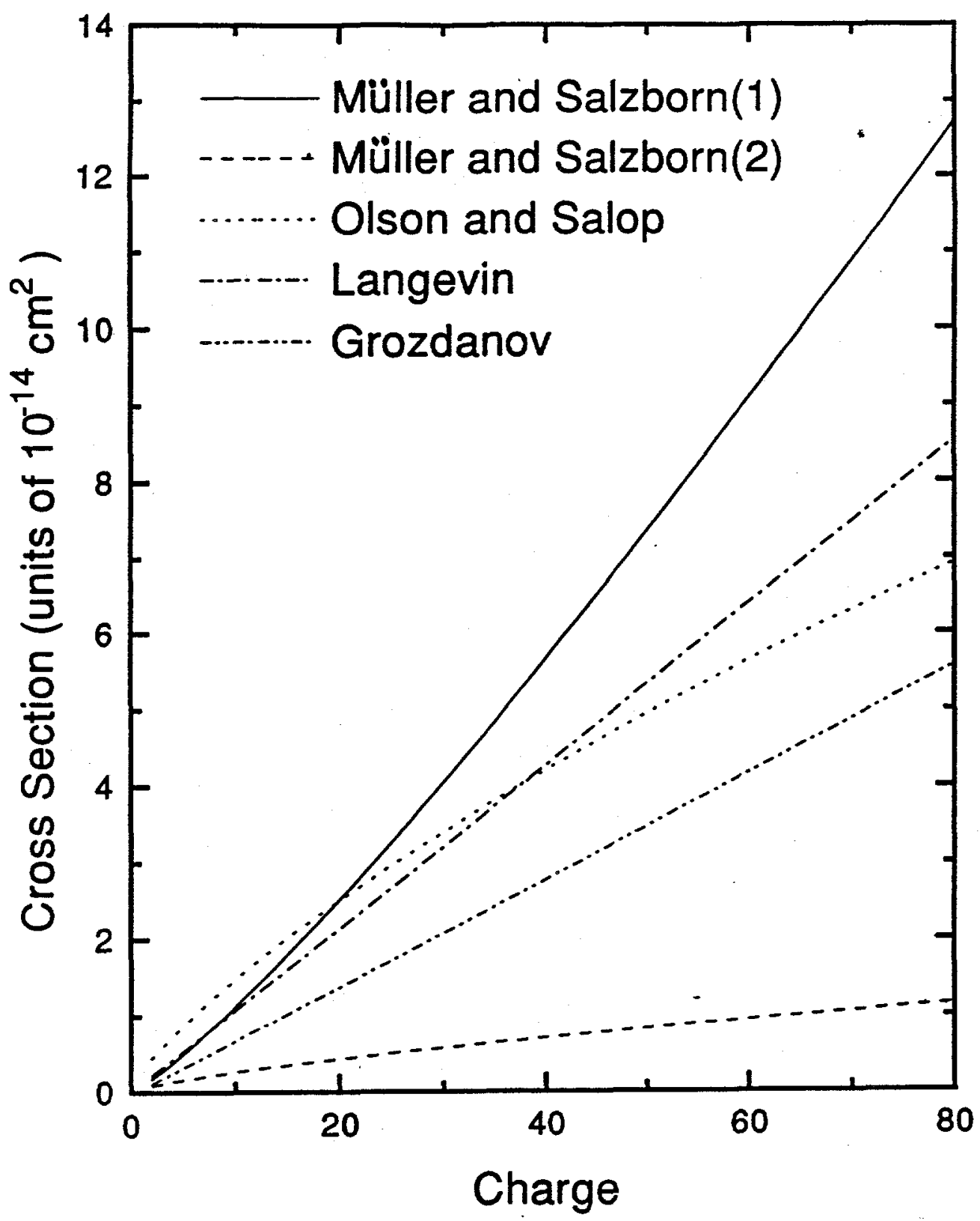

Figure 3.2: Theoretical electron capture cross-section vs. charge for heavy ions and molecular hydrogen. Energy in the center of mass frame is $2 \mathrm{eV}$. 
where $\mathrm{R}$ is the internuclear separation. By evaluating the classical turning points along the $\xi$ and $\eta$ axes, it was discovered that the electron is constrained to move around only one center when the internuclear separation $R$ is greater than a critical distance $R_{0}$ and can move around both centers when the internuclear separation is less than $R_{0}$ where

$\mathrm{R}_{0}=2(2 \mathrm{z}-1)^{1 / 2}$

in units of $\mathrm{a}_{0}$. An energy dependence appears in the charge transfer cross section via a probability per unit time that the electron will transfer between cores while the internuclear separation is less than $R_{0}$. However, at impact energies less than about 0.5 $\mathrm{keV} / \mathrm{amu}$ (still much higher than the energies of interest in RETRAP experiments) charge transfer probability is approximately equal to one in the classically allowed region, so cross section is energy independent and given by

$\sigma_{\mathrm{G}}=4 \pi(2 \mathrm{z}-1)$

in units of $\mathrm{a}_{0}^{2}$.

Although Grozdanov's treatment was for fully stripped ions and atomic hydrogen, the shell structure of the ion should not matter since at the distance $\mathbf{R}_{\mathbf{0}}$ calculated above the ion looks like a point charge. As a first approximation it is probably reasonable to treat the hydrogen molecule as one electron orbiting a point charge at the molecular center, since $\mathrm{R}_{0}$ is large compared to the size of a hydrogen molecule. The cross sections shown in the figures are the values for atomic hydrogen.

This classical approximation is expected to be a lower bound to the true cross section, since the electron can tunnel when charge exchange is classically forbidden.

Now we consider the "absorbing sphere" model of Olson and Salop [9]. Before charge exchange there is a small attractive force due to the induced dipole moment of the hydrogen molecule. After charge exchange there is a strong repulsive force. Charge exchange is assumed to occur at one of the points where the potential energy curves with 
and without electron transfer cross. Since there are many excited states the electron may be captured into, there are many such crossings. Olson and Salop concluded that for internuclear distances greater than a critical distance $R_{c}$ the probability of electron transfer was negligibly small, while for distances less than $R_{c}$ charge transfer probability was nearly one. Thus, the cross section $\sigma$ is simply

$\sigma=\pi R_{c}^{2}$

The equation which determines $R_{c}$ was found semiempirically,

$R_{c}^{2} \exp \left[-2.648 \alpha R_{c} / z^{1 / 2}\right]=2.864 \times 10^{-4} z(z-1) v / f$

with $\alpha=\left(I_{t} / 13.6\right)^{1 / 2}$ where $I_{t}$ is the ionization potential of the target molecule in $\mathrm{eV}$ (the ionization potential of $\mathrm{H}_{2}$ is $15.4 \mathrm{eV}$ ), $\mathrm{v}$ is the relative velocity, and $\mathrm{f}$ is the Franck-Condon factor for specific transitions between vibrational levels of the target molecule. For monatomic targets, $\mathrm{f}$ is replaced by one. In the diagrams the value used for the Franck-Condon factor is 0.1 . This value was obtained from Figure 5 of Olson and Salop's paper. However, the dependence of $R_{c}$ on $f$ is very weak. Since $f$ and $v$ only enter equation 2.4 as the ratio $v / f$, the dependence of $R_{c}$ on $f$ can be immediately seen from the dependence of $R_{c}$ on energy. Note that this equation has multiple solutions. It is the higher positive value of $R_{c}$ which is physically meaningful. The authors consider this model applicable for relative velocities $<10^{8} \mathrm{~cm} / \mathrm{sec}(10 \mathrm{keV}$ in the center of mass frame for $\mathrm{H}_{2}$ ).

For very low energies (less than $1 \mathrm{eV}$ in the center of mass frame) the Langevin model [10] should be more appropriate. As noted above, the ion will induce a dipole moment in the neutral. The neutral thus effectively moves in an inverse fourth order potential. Orbits in such a potential are unstable, and it is found that if the atom has an impact parameter $b$ less than some critical impact parameter $b_{0}$ the neutral and the ion will spiral toward their mutual center of mass until charge exchange takes place. If 
charge exchange never takes place at a radius greater than $b_{0}$ the charge exchange cross section will be

$\sigma=\pi b_{0}^{2}$

$b_{0}$ is given by

$b_{0}=\left(4 z^{2} \alpha_{D} / \mu v^{2}\right)^{1 / 4}$

where $\mu$ is the reduced mass, $\alpha_{D}$ is the polarizability of the neutral, and $v$ is the relative velocity. The polarizability $\alpha_{\mathrm{D}}$ of $\mathrm{H}_{2}$ is $5.3 \mathrm{a}_{0}^{3}\left(8.0 \times 10^{-25} \mathrm{~cm}^{3}\right)$. Note that, because the cross section is inversely proportional to velocity, the charge exchange rate $\mathrm{k}$ is energy independent (see eqn 1.1). This strong velocity dependence is an exception to the principle that charge transfer cross sections should be weakly energy dependent because the actual radius at which charge transfer occurs is irrelevant to the Langevin cross section.

Finally we consider an empirical scaling law developed by Müller and Salzborn [11]. The law was developed from collisions with energies ranging from about $10 \mathrm{keV}$ to $100 \mathrm{keV}$. Cross sections were seen to be nearly energy independent in this range. The scaling law is

$\sigma=A z^{j} I^{k}$

where $z$ is the ion charge, $I$ is the first ionization potential of the neutral, and $A, j$, and $k$ are fitting parameters with the values $A=5.6$ (in units of $a_{0}{ }^{2}$ ), $j=1.17$, and $k=-$ 2.76. Müller and Salzborn use the same fit function for multiple electron capture (up to 4 electrons captured). For double capture $A=3.7, j=0.71$, and $k=-2.8$. The first ionization potential was used for any number of electrons captured, because using the $\mathrm{nth}$ ionization potential for capturing $\mathrm{n}$ electrons was found not to improve the fit. 
When two electrons are captured, the ion is initially in a double excited state. The ion may radiatively stabilize (called "true double capture"), or may autoionize through an Auger process (called "transfer ionization"). In the experiments fitted above (and also in RETRAP experiments), charge exchange rates are calculated by measuring charge exchange products, thus transfer ionization is indistinguishable from (and is counted as) single capture. Theoretical calculations [12] give as a ratio for the rates of Auger to radiative decay

$\mathrm{W}_{\mathrm{a}} / \mathrm{W}_{\mathrm{r}} \cong\left(10^{4} / \mathrm{z}^{4}\right)\left(\mathrm{I}_{1} / \mathrm{I}_{2}\right)^{3 / 2}$

where $I_{1}$ and $I_{2}$ are the first and second ionization potentials of the neutral. Since the Müller and Salzborn fit results were based on incident ions with charge $\leq 10$, many double captures were followed by autoionization, whereas for $Z>20$ almost all double captures should be radiatively stabilized. However, experimental results have contradicted this [13], showing high rates of autoionization for $Z$ up to $44+$. A recent review of the study of multiple electron capture by highly charged ions may be found in [14]. 


\section{CHAPTER IV}

\section{EXPERIMENTAL METHOD}

\section{A. The Electron Beam Ion Trap (EBIT)}

The production of highly charged ions in EBIT (Electron Beam Ion Trap) is described in detail in [15]. Briefly, EBIT contains three cylindrical "drift tubes" held at high potential $(500 \mathrm{~V}-30 \mathrm{kV})$ and an electron gun held near ground. Current from the electron gun can be varied from zero to about $150 \mathrm{~mA}$ by varying the voltage on the anode. A "bucking coil" holds the magnetic field at the electron gun near zero. The electron beam is compressed by a 3 Tesla superconducting magnet, to a current density of about $4 \mathrm{kA} / \mathrm{cm}^{2}$ at maximum current. Neutrals or low charge state ions are successively ionized by electron impact. The "top" and "bottom" drift tubes are held at a slightly higher voltage than the "middle" drift tube, thus the ions are confined axially. The ions are confined radially by the electric field of the electron beam and by the magnetic field. Most of the confining force comes from the electron beam.

Target elements are injected into EBIT in several ways. Gasses are injected as neutrals by a gas injector mounted on the side of EBIT. Metals are injected as low charge state ions ( +1 to +4 ) created in a Metal Vapor Vacuum Arc (MeVVA) [16] mounted above EBIT. Rare isotopes may be inserted as neutrals using a wire probe [17].

\section{B. Ion Extraction and Transport}

Ions are extracted from EBIT by increasing the voltage on the middle drift tube with a linear voltage ramp. Since the ions escape when their axial kinetic energy is just enough to exceed the potential barrier caused by the top drift tube, all ions of a given charge state will have nearly the same axial energy. Equivalently, all ions see the same 
accelerating potential from rest. Such axial energy spread that exists is due to fluctuations of the energy of the top drift tube or the high voltage platform (less than 5 volts), or to the energy gained during one round trip through the center drift tube. The latter includes energy gained in ion-ion or ion-electron collisions and the energy gained by the rising potential of the middle drift tube. This last term is given by

$\mathrm{U}=\mathrm{T}_{\mathrm{t}}\left(\mathrm{V}_{\mathrm{r}} / \mathrm{t}_{\mathrm{r}}\right)$

where $T_{t}$ is the round trip time for an ion (estimated to be about 2 microseconds), $V_{r}$ is the voltage change during the ramp, and $t_{r}$ is the rise time of the ramp.

Because the maximum number of ions which can be captured in RETRAP is the number that are in RETRAP at the same time, it was necessary to radically increase the ramp rate in order to capture a sufficient number of ions.

In "slow extraction" mode (used for ion-surface interaction experiments), $V_{r}$ is about 200 volts and $t_{r}$ is about 100 milliseconds, resulting in a spread in accelerating potential of $4 \mathrm{mV}$ (negligible). Further, the extraction time is long compared to the ionion collision time (estimated to be 100 microseconds) [18]. The ions which escape will be those with the highest axial energy, thus the remaining ions will be cooled. This effect decreases the average transverse energy. Transmission efficiency is up to 90 percent, with about $10^{4}$ to $10^{5}$ ions of a given charge state per pulse (depending on the particular ion desired) delivered to a target.

In "fast extraction" mode (developed for RETRAP experiments) $V_{r}$ is still about 200 volts but $t_{r}$ is about 10 microseconds, leading to a spread in accelerating potential of about 40 volts. The extraction time is short compared to the ion-ion collision time, so there is no appreciable cooling of the transverse energy. The higher spread of energy is assumed to be the cause of the decrease in transport efficiency. Typically about 200 ions per pulse are detected below RETRAP.

After ejection from EBIT the ions are focused by an einzel lens, bent 90 degrees by an electrostatic bender, and charge state selected by a 90 degree magnet. The 
selected ions pass through two more 90 degree electrostatic benders before entering the RETRAP chamber. The straight sections of beam tube (except the short section between the magnet and the second electrostatic bender) each contain an einzel lens approximately at the midpoint. These lenses ensure that the beam diameter is always a minimum at each bender.

A second MeVVA is mounted directly above RETRAP, allowing for the injection of large numbers $\left(\sim 10^{5}\right)$ of singly or doubly charged ions. This was used to test RETRAP apparatus independent of EBIT, and to estimate the neutral particle density in RETRAP. A schematic of the EBIT-RETRAP system is shown in Figure 4.1.

It is important to note that all positive ions with a velocity proportional to $(q / m)^{1 / 2}$ (which includes ions accelerated from rest) and acted upon only by electric fields with no time variance will follow the same geometric path. This can easily be demonstrated by introducing a variable $t^{\prime}=t(q / m)^{1 / 2}$. The acceleration due to an electric field $E$ is

$\mathrm{d}^{2} \mathrm{x} / \mathrm{dt^{ \prime 2 }}=\mathrm{E} / 4 \pi \varepsilon_{0}$

so the equations of motion have no $\mathrm{q} / \mathrm{m}$ dependence.

The charge selection magnet may be treated (approximately) as a square region of constant magnetic field surrounded by a region of no magnetic field. The ions of the charge state selected will follow a cyclotron orbit with a radius $r_{m}$ which is a characteristic length of the magnet. Since the force on the ions is given by

$F_{B}=q v B=m v^{2} / r_{m}$

and $\mathrm{v}=\left(2 \mathrm{qV} \mathrm{V}_{\mathrm{A}} / \mathrm{m}\right)^{1 / 2}$ for a constant accelerating voltage $\mathrm{V}_{\mathrm{A}}$,

we have 


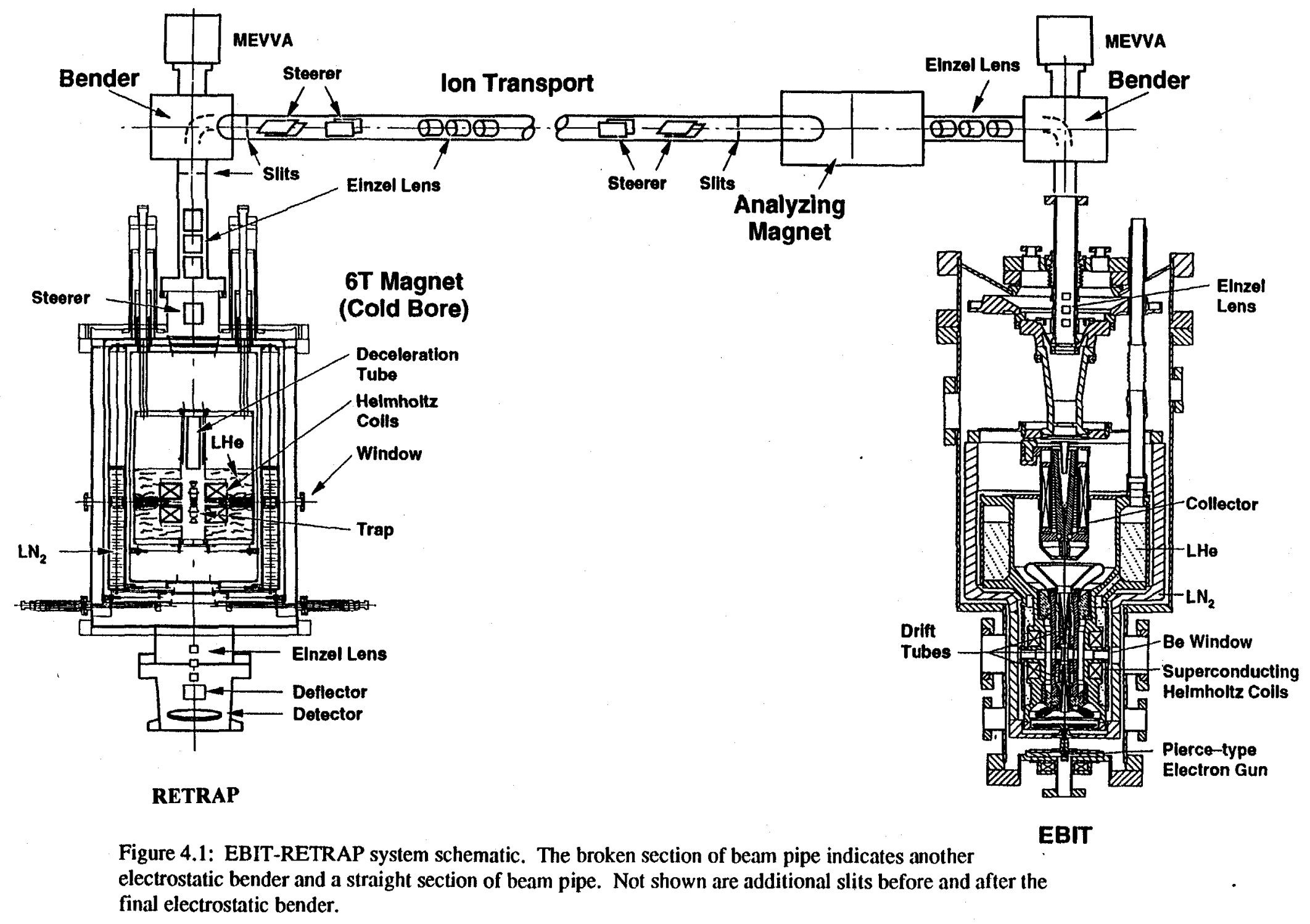


$B=\left(1 / r_{m}\right)\left(2 V_{A} m / q\right)^{1 / 2}$

or, equivalently,

$r_{m}=(1 / B)\left(2 V_{A} m / q\right)^{1 / 2}$

The field in the electrostatic benders is not uniform. Approximating the benders as complete cylinders of infinite length, the radial electric field at a distance $r$ from the center of curvature is

$E_{r}=V_{B} /\left(r \ln \left(r_{0} / r_{i}\right)\right)$

where $V_{B}$ is the potential difference between the benders and $r_{o}$ and $r_{i}$ are the inner and outer radii of the cylinder segments (about $6 \mathrm{~cm}$ and $4 \mathrm{~cm}$ respectively). For circular ion paths the force due to the electric field is the centripetal force,

$\mathrm{F}_{\mathrm{E}}=\mathrm{qE}_{\mathrm{r}}=\mathrm{mv}^{2} / \mathrm{r}$

Combining these equations yields

$V_{B}=V_{A} \ln \left(r_{0} / r_{i}\right)$.

The absence of an $r$ dependence in this equation indicates that all circular ion paths for a given charge state require the same velocity.

A first order treatment of charged particle optics, including dispersion effects for a spread in energy, is found in [19]. Briefly, The focal length of a cylindrical bender of angle $\Phi$ and central radius $r_{e}$ is given by 
$f=\left(r_{d} / 2^{1 / 2}\right) / \sin \left(2^{1 / 2} \Phi\right)$

which for our 90 degree bender gives a focal length of $0.89 \mathrm{r}_{\mathrm{e}}$. This focusing is in one dimension only; there is no focusing in the dimension parallel to the cylinder axis.

Thus, these deflectors always introduce astigmatism into the beam. However, minimizing the spot size at each bender also minimizes this effect.

The energy dispersion coefficients $K$ and $L$ are defined by

$\mathrm{y}_{2}=\mathrm{K} \gamma+\mathrm{y}_{1}$

$$
\mathrm{y}_{2}^{\prime}=\mathrm{L} \gamma+\mathrm{y}_{1}^{\prime}
$$

where $y_{2}$ is the displacement from the central ion path exiting the optical element, $y_{1}$ the displacement entering the element, $y_{2}^{\prime}$ the slope relative to the central ion path exiting the element, $y_{1}^{\prime}$ the slope entering the element, and $\gamma$ the energy dispersion defined by

$\gamma=\left(\mathrm{U}-\mathrm{U}_{0}\right) / \mathrm{U}_{0}$

where $U$ is the energy of a particular ion and $U_{0}$ is the average ion energy. For the electrostatic bender $K=.80 r_{e}$ and $L=.56$. For the magnet $K=r_{m} / 2$ and $L=1 / 2$. Based on eqn (4.1), $\gamma$ has a maximum value of about .005 . Since $r_{e}$ is about $4 \mathrm{~cm}$ and $r_{m}$ is about $20 \mathrm{~cm}$, the energy dispersion causes a maximum of $.05 \mathrm{~cm}$ displacement and .0028 increase in slope in each element. Since the distance between elements tends to be about 1-2 meters and the effective aperture for capture in RETRAP is probably less than $1 \mathrm{~mm}$, energy dispersion effects are probably a significant cause of the low transport efficiency of fast extraction. 
C. Ion Capture

Just before entering RETRAP the ions pass through a two segment "deceleration tube," each segment about $12 \mathrm{~cm}$ long. The magnetic field is less than 0.1 Tesla at the top of the deceleration tube and is nearly full field at the bottom. The tube is typically biased at about $3.5 \mathrm{kV}$. As the ions are passing through, the tube voltage is quickly (less than $50 \mathrm{~ns}$ ) pulsed down by nearly the accelerating voltage. The ions emerge with an energy range of $0-100(q / e) e V$. The deceleration tube voltage is controlled by a circuit built around a Lasermetrics GS-9 pulser. A circuit schematic is shown in Figure 4.2 .

Before the ions emerge from the deceleration tube, the top electrode of RETRAP is pulsed down to zero volts to allow the ions to pass through. The ions are reflected off the bottom electrode of RETRAP. The voltage of the top electrode is then pulsed up. The number of ions which may be trapped is limited to the number of ions which emerge from EBIT during a time equivalent to an ion round trip time in RETRAP (about 0.8 microseconds).

Another lower bound on the number of ions which may be trapped in a cycle is the number of ions traveling through the deceleration tube at a given time. Assuming the ion pulse from EBIT is too long to trap the entire pulse (this has always been the case in practice), ion density in the deceleration tube should be inversely proportional to the velocity,

$\mathrm{N} / \mathrm{L} \sim\left(\mathrm{V}_{\mathrm{db}}-\mathrm{V}_{\mathrm{a}}\right)^{-1 / 2}$

where $\mathrm{V}_{\mathrm{db}}$ is the deceleration tube bias and $\mathrm{V}_{\mathrm{a}}$ is the accelerating potential. From this relation it would appear that the deceleration tube bias should be nearly equal to the accelerating voltage to maximize the number of trapped ions. Unfortunately, the ions 


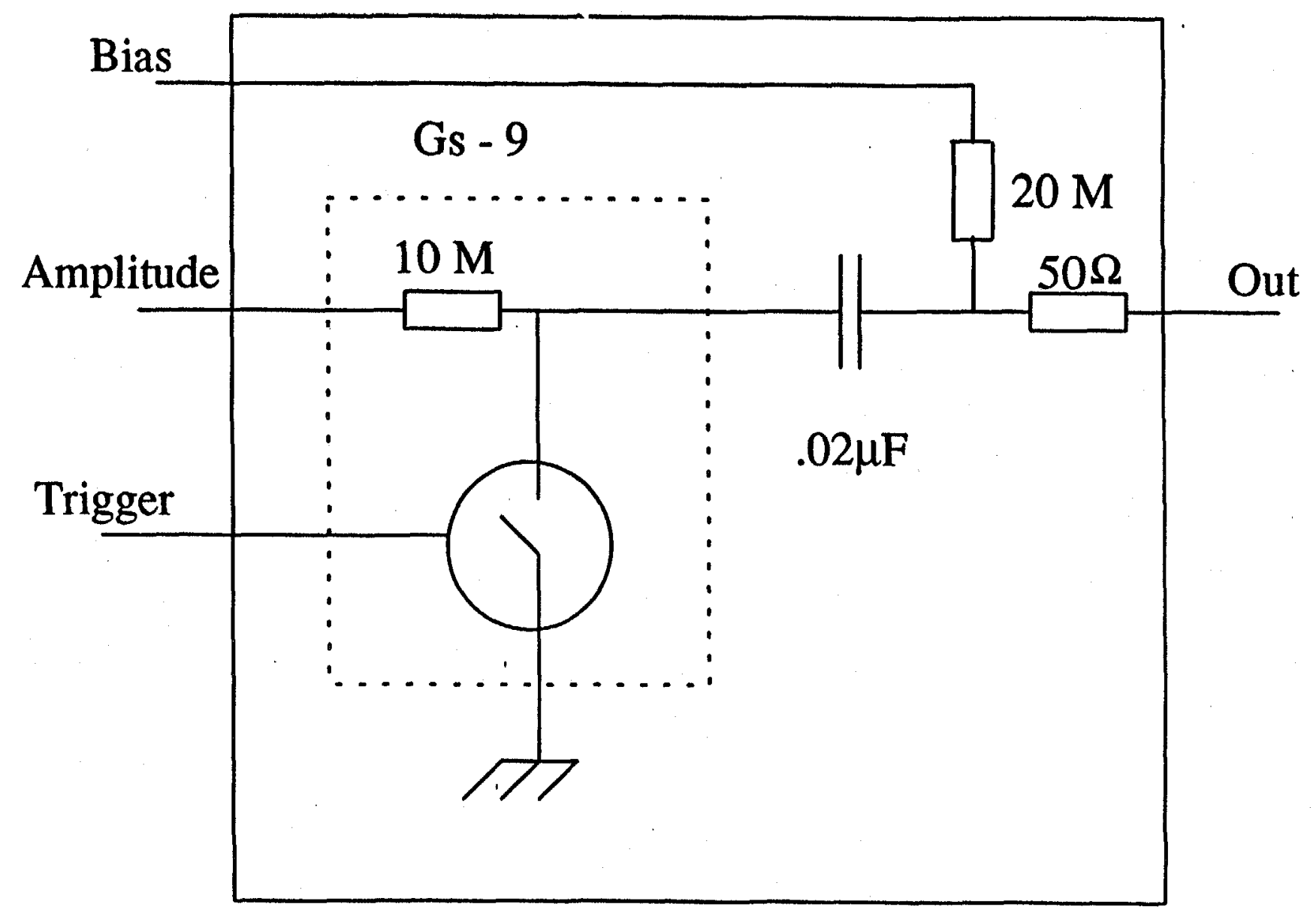

Figure 4.2: Deceleration tube circuit schematic. The circled area represents a thyratron switch. 
are so strongly focused upon entering the deceleration tube, the focal point is inside the deceleration tube. Increasing $\mathrm{V}_{\mathrm{db}}$ decreases the focal length.

As the ions approach the trap, their motion is increasingly affected by the magnetic field. For approximately the bottom $6 \mathrm{~cm}$ of the deceleration tube, the magnetic field changes only slightly during a cyclotron orbit (i.e. the motion becomes adiabatic) and the quantities $\mathrm{Br}_{\mathrm{c}}{ }^{2}$ and $\mathrm{v}_{\mathrm{t}}{ }^{2} / \mathrm{B}$ become invariant, where $\mathrm{r}_{\mathrm{c}}$ is the cyclotron radius and $v_{t}$ is the velocity transverse to the magnetic field. Since total kinetic energy is conserved, the result is a transfer from axial energy to transverse energy. Note that the magnetic field lines are converging in the deceleration tube, so an off axis ion converging toward the axis may have a lower $v_{t}$ than an ion traveling parallel to the axis. Thus, increasing $\mathrm{V}_{\mathrm{db}}$ increases ion density but can also increase the spread in axial energy. The optimum voltage for trapping was found empirically.

The number of ions in the tube is also, of course, proportional to the tube length. However, a longer tube may have a lower optimum $\mathrm{V}_{\mathrm{db}}$ because the magnetic field is weaker and is converging more slowly at the top of a longer tube. Experimentally, it was found that pulsing both $12 \mathrm{~cm}$ deceleration tube segments together yielded about the same number of ions captured as holding the top segment at a fixed voltage and pulsing the bottom segment, although the parameters for optimum trapping efficiency were different.

The pulsing on the endcap electrodes is accomplished using Avtec AVR-3 pulsers. The pulsers produce a negative pulse of up to $200 \mathrm{~V}$ from zero volts with duration controlled by the length of an input pulse and amplitude controlled by a potentiometer on the front panel. It was necessary to "float" the pulsers in order to achieve a pulse from a positive value to zero volts. Also, the pulsers constantly produce small fluctuations on the voltage at the pulser output. Capacitive coupling between the endcap and compensation electrodes caused interference on the preamp signal (see detection). It was necessary to decouple the pulsers from the endcap electrodes (using relays) except during the time the pulsers were actually pulsing. A schematic of the 
pulser setup is shown in Figure 4.3. The same type of circuit was used for both endcap electrodes (to "catch" and "dump" the ions).

The ions were detected using electron multipliers or microchannel plate detectors, amplified by Ortec VT120 preamplifiers mounted just outside the vacuum system and further amplified by Ortec 579 amplifiers, pulse height discriminated by Ortec 436 discriminators, and counted using Lecroy 2551 or Kinetic Systems 3615 scalers. The nominal maximum count rate of the system is $100 \mathrm{MHz}$ (a faster system is planned for future experiments). Ion flux from EBIT exceeds this (a detector placed before the charge selection magnet saturates), and ion flux on the detector below RETRAP was sufficiently high to cause some decrease in detection efficiency.

Optimization of parameters was done in three steps. First, with the deceleration tube grounded and all trap pulsing disabled, transport parameters were adjusted to maximize counts on the detector below RETRAP. Second, trapping was enabled with ions held a short time ( $\sim 1$ second), then dumped. Trapping parameters were adjusted to maximize the number of dumped ions. Finally, beam transport parameters were adjusted

again to maximize the number of dumped ions. Since trapping efficiency was $\sim 10 \%$, saturation effects were not a problem for the dumped ions.

In the experiments described here the "catch" and "dump" circuits were identical in order to keep the voltages on the top and bottom electrodes as nearly symmetrical as possible. The voltage on the top electrode must be changed quickly in order to trap ions; however, in priciple the voltage on the bottom electrode could be lowered arbitrarily slowly. In some tests of the apparatus, the bottom electrode pulser was replaced by a slow ramp generator to gain information on the energy distribution of the stored ions, or to avoid saturation on the bottom detector (when using the MeVVA).

With the number of captured ions optimized, ion storage time was increased (typically to $\sim 200$ seconds) and lifetime measurements could be performed. 


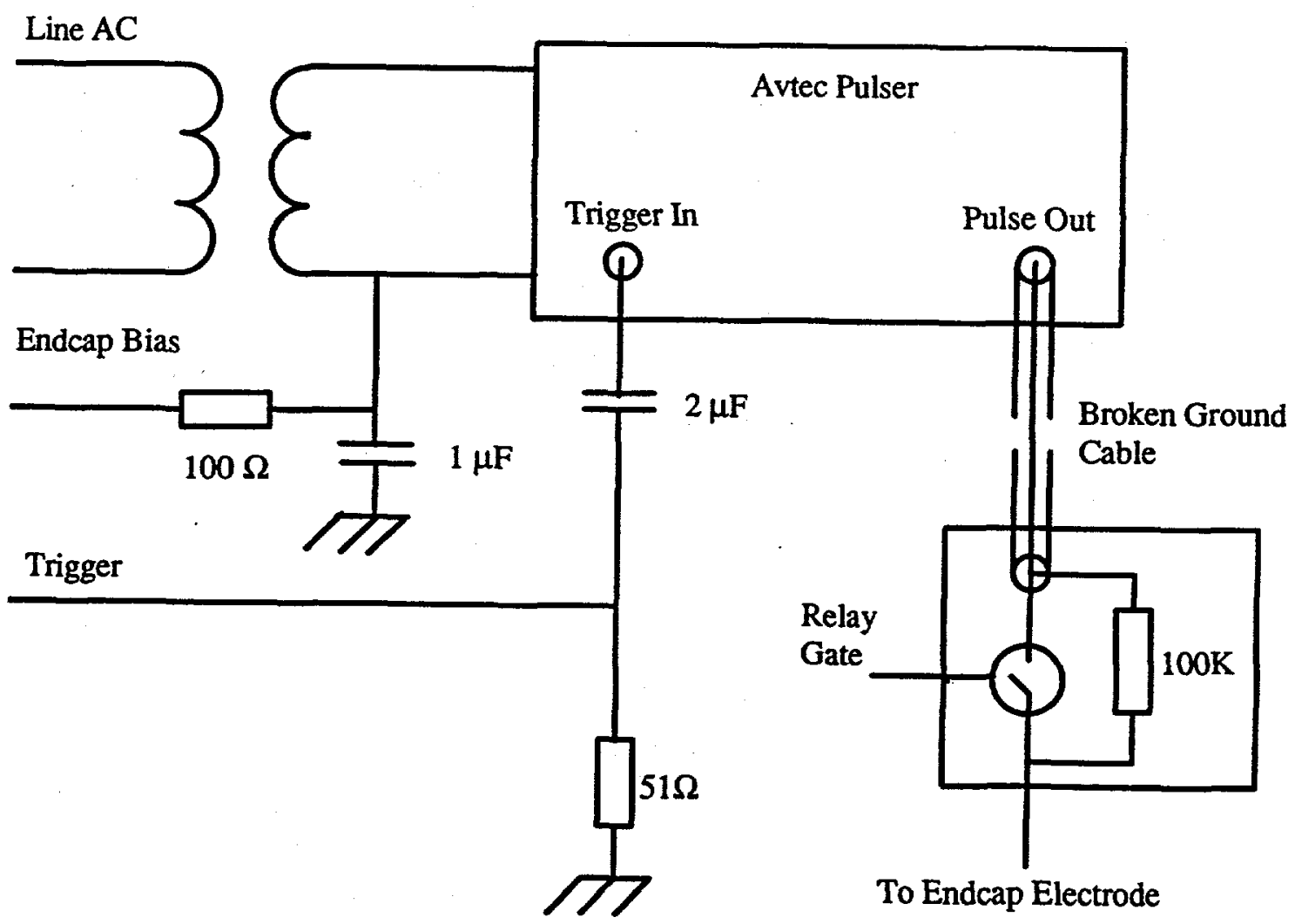

Figure 4.3: Endcap electrode circuit schematic. The "broken ground cable" is a coaxial cable with the shield cut through to allow a potential difference between ground and "zero volts" as seen by the Avtec pulser. 
D. The Penning Trap

The electric potential near the center of a Penning trap can be expanded in Legendre polynomials,

$V=(1 / 2) V_{0} \sum_{k} C_{k}(r / d)^{k} P_{k}(\cos \theta)$

where $d$ is a characteristic dimension of the trap defined by $d^{2}=\left(z_{0}^{2}+\rho_{0}^{2} / 2\right) / 2$ where $p_{0}$ is the radius and $z_{0}$ is the length of the compensation electrode plus half the length of the middle electrode (see Figure 4.4).

For any symmetric trap the odd terms will all be zero. For an ideal trap all terms except $\mathrm{C}_{0}$ and $\mathrm{C}_{2}$ are zero and the magnetic field $\mathrm{B}$ is uniform in magnitude and directed along the trap axis of symmetry. Since $\mathrm{C}_{0}$ adds a constant potential over the entire trap, it has no effect on ion motion. Ions with mass $\mathrm{m}$ and charge $\mathrm{q}$ oscillate with an axial angular frequency of

$\omega_{z}^{2}=\left(q V_{0} C_{2}\right) /\left(m d^{2}\right)$

and execute radial motions with angular frequencies given by

$\omega_{+}^{2}=(1 / 2)\left(\omega_{c}+\left(\omega_{c}^{2}-2 \omega_{z}^{2}\right)^{1 / 2}\right)$

$\omega_{.}^{2}=(1 / 2)\left(\omega_{c}-\left(\omega_{c}^{2}-2 \omega_{z}^{2}\right)^{1 / 2}\right)$

where

$\omega_{\mathrm{c}}=\mathrm{qB} / \mathrm{m}$ 


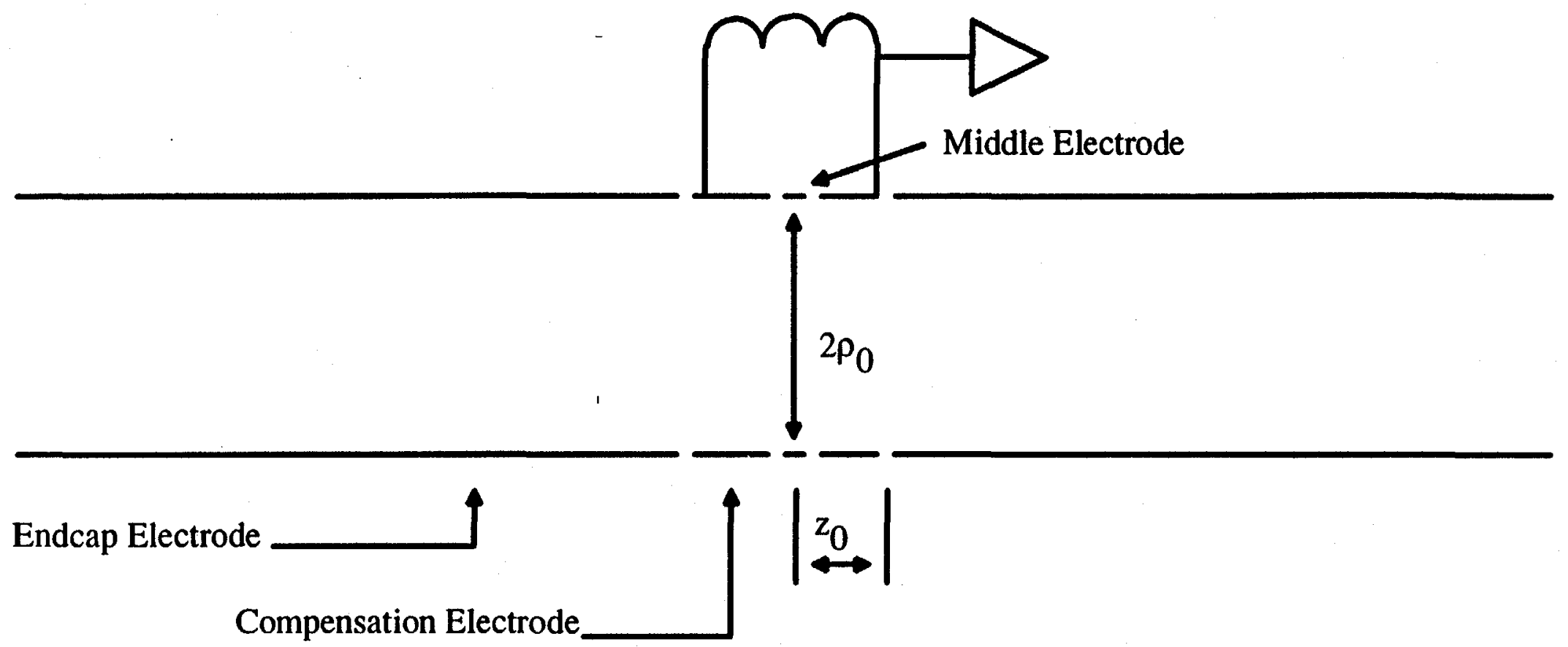

Figure 4.4: The Penning trap. An external inductor attached to the two compensation electrodes in parallel with stray capacitances forms a tuned circuit. The characteristic length $d$ of the trap is defined by $d^{2}=\left(z_{0}^{2}+p_{0}^{2} / 2\right) / 2$ 
is the unperturbed cyclotron angular frequency. $\mathrm{C}_{2}$ is a dimensionless constant depending on trap geometry (equal to 0.5449 in RETRAP). $\omega_{+}$is the perturbed cyclotron angular frequency, $\omega_{-}$is the magnetron angular frequency. A review of the physics of Penning traps is found in [20].

Because the equipotential surfaces are hyperboloids, Penning traps are often constructed with hyperbolic electrodes. However, such traps are still imperfect because the electrodes must be truncated at some point, traps require slits or holes to allow charged particles or light to enter, and in any case there will be machining imperfections. RETRAP is constructed as a series of five open-ended coaxial cylinders, following a design similar to that used by Gabrielse et al. [21]. The potential difference between the endcap electrodes and the middle electrode determines the trap depth. Adjusting the compensation electrodes allows us to cancel out the lowest order anharmonicity term (the $\mathrm{C}_{4}$ term). In RETRAP the optimum voltage on the compensation electrodes is about 0.12 times the endcap voltage. Machining imperfections are much smaller in a cylindrical than a hyperbolic trap. Calculating the internal potentials is also much easier than for a real (truncated) hyperbolic trap. The open ends allow for relatively easy injection of ions. Unfortunately, anharmonic effects are greater in the cylindrical trap. Figure 4.5 shows the potential along the axis of RETRAP. Figure 4.6 shows the ion axial frequency as a function of position for ions in RETRAP, holding potentials on the electrodes fixed.

Magnetic field homogeneity is about 30 parts per million over the region in which ions are trapped.

The compensation electrodes are connected by an inductor with an inductance $\mathrm{L}$ of about 700 microhenrys. The inductor and the stray capacitances between the two compensation electrodes forms a tuned circuit with a resonance frequency $f_{0}=\omega_{0} / 2 \pi$ of about 1.22 megahertz and a quality factor $Q$ of about 200 (cold). The image current induced by the ions on the compensation electrodes is amplified by a GaAs preamplifier (similar to one designed by A. Lee [22]) bolted to the bottom of the helium dewar. The preamplifier was mounted in a stainless steel box sealed with gold wire in order to isolate 


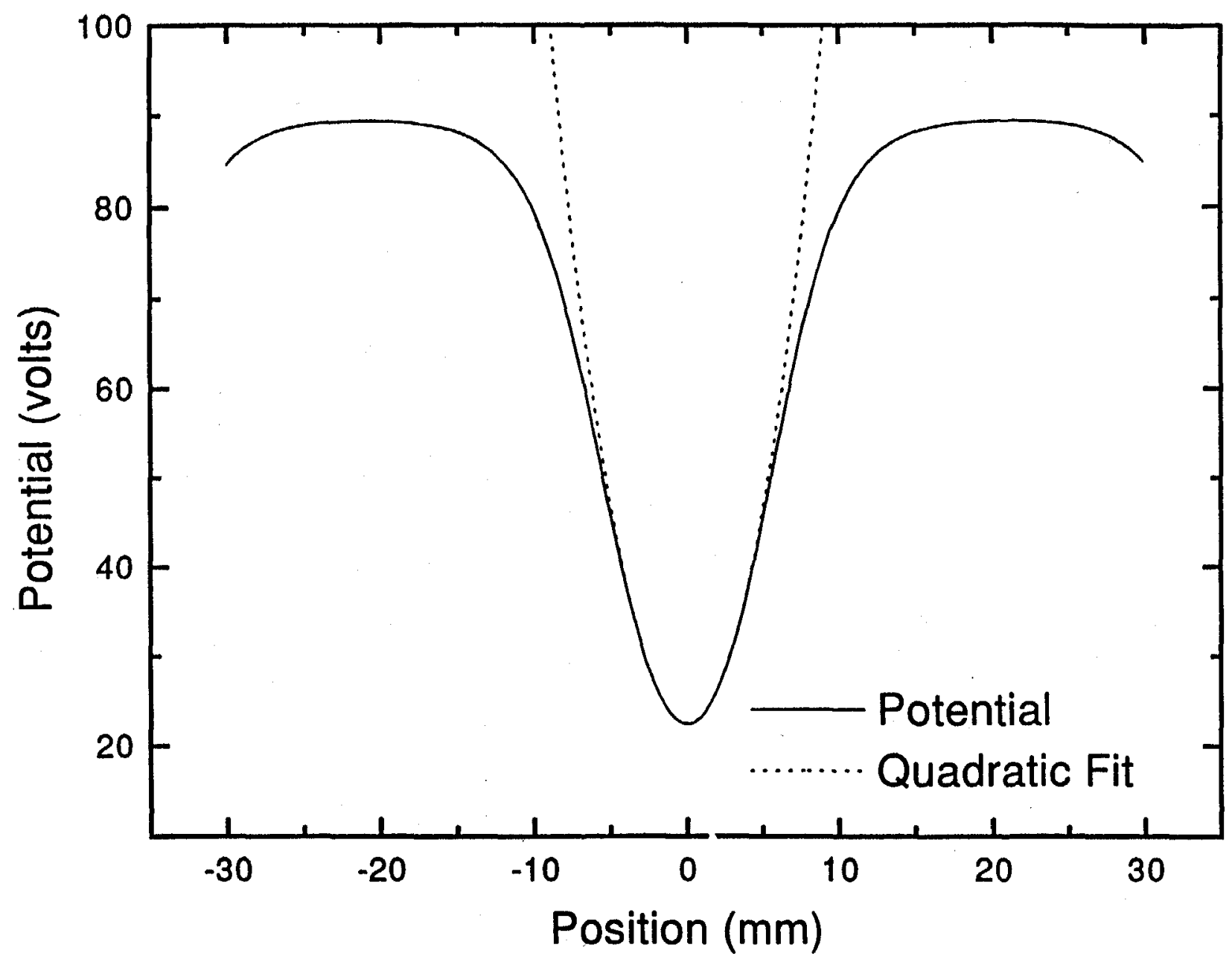

Figure 4.5: Potential along the trap axis. The potential rises less steeply than a pure quadratic, so higher amplitude ions have a lower frequency. 


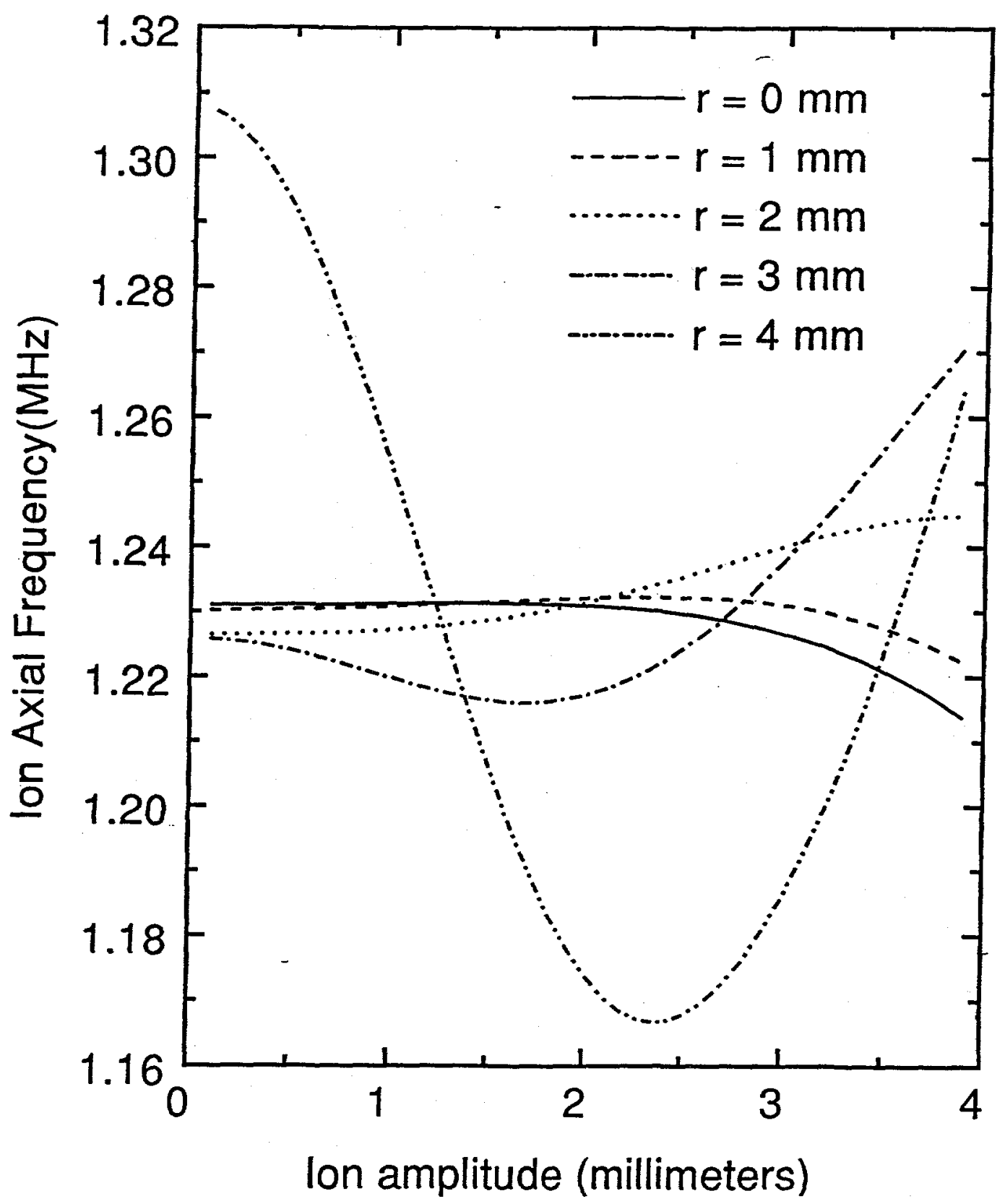

Figure 4.6: Frequency dependence on ion amplitude and radius. 
it from the RETRAP vacuum. The preamplifier has an input impedance of about 1.5 $\mathrm{M} \Omega$, an output impedance of about $50 \Omega$, and a high frequency $3 \mathrm{~dB}$ rolloff of about 30 Mhz. Although the magnetic field at the position of the preamplifier is about 2 Tesla, the preamplifier has been tested (warm) in the center of the magnet and can operate in a field of 6 Tesla.

The signal from the preamplifier is further amplified outside the chamber. This signal is then observed on an HP 3589A Spectrum/Network Analyzer. The spectrum analyzer can be operated in "swept frequency" or "zero span" mode.

In swept frequency mode the spectrum analyzer displays amplitude vs. frequency over some range. This mode was used to find the center and the $Q$ of the tuned circuit. When no ions are in the trap, the thermal noise at the input of the preamplifier is multiplied by the frequency response of the tuned circuit. The spectrum analyzer shows a peak at the tuned circuit resonance, and the $Q$ is given by

$Q=f_{0} / \Delta f$

where $f_{0}$ is the frequency of the resonance and $\Delta f$ is the full width at half maximum power of the peak.

In zero span mode the spectrum analyzer displays the amplitude within a fixed frequency range versus time. It is in this mode that ion signals were measured.

Information on the population of various ion charge states was found by adjusting the endcap electrode voltages to bring the ion axial oscillation frequency into resonance with the tuned circuit. Frequencies of different low amplitude ions of the same charge state in RETRAP were found to be the same to about 1 part in 1000, leading to a coherence time of about 1 millisecond. Since observations times were always much longer than this, ion signals added incoherently, so the measured signal was proportional to an average ion amplitude times the square root of the number of ions. 
The image currents which the ions induce cause them to lose energy. The one dimensional cooling time constant is

$t_{z}=4 m d^{2} / q^{2} R_{\text {eff }}$

where $R_{e f f}$ is the effective resistance. On resonance $R_{e f f}=R_{0}$ is the effective parallel resistance of the tuned circuit

$\mathrm{R}_{0}=\mathrm{Q} \omega_{0} \mathrm{~L}$

which is about $1.5 \mathrm{M} \Omega$ in RETRAP. Off resonance the effective resistance is given by

$R_{\text {eff }}=R_{0} /\left(1+Q^{2}\left(\omega / \omega_{0}-\omega_{0} / \omega\right)^{2}\right)$.

Because of the large $Q^{2}$ factor in the denominator, cooling effects are generally negligible for ions off resonance.

E. Measurement of Ion Signals

Before capturing a pulse of ions from EBIT, the endcap electrodes were set such that stored ions would oscillate at a frequency somewhat below the tuned circuit frequency. For example, it was determined that the proper endcap voltage for bringing $\mathrm{Xe}^{44+}$ ions on resonance was 90 volts. The ions were captured with an endcap voltage of about 70 volts. The ions were held off resonance for a certain time, then brought on resonance. By measuring the signal after various times, charge exchange rates were inferred.

To detect the ions, the endcap voltages were increased with a linear ramp of about 40 volts over 2 seconds, then quickly brought down again. As the ramp voltage increases, the axial frequency of the ions increased and they were successively brought 
onto resonance. Figure 4.7 shows an output spectrum just after injection. The broad peak is the signal as the ions come onto resonance as the ramp goes up, the narrow peak as the ramp comes down.

The width of the peak is determined by the frequency distribution of ions in the trap and the quality factor of the tuned circuit. For ions near the trap axis, the higher the amplitude of the axial motion of ions, the higher the voltage must be to bring the ions on resonance (see Figure 4.6). It was discovered that by adiabatically decreasing the endcap voltages in order to allow the higher energy ions to escape ("predumping"), the peak width of an individual charge state could be decreased considerably (Figure 4.8). Figures 4.7 and 4.8 have the same horizontal scale, and both represent only the injected state $\left(\mathrm{Xe}^{44+}\right)$. Each represents the averaged signal of about 30 injection cycles, or roughly 100 ions.

As the voltage on the endcaps decreases during the predump, the ions cool. The action $\mathrm{J}$ defined by

$J_{Z}=\oint p_{z} d z$

is a conserved quantity (so long as the voltage change is adiabatic). For ions still in a harmonic region of the trap, this implies the quantity $A^{2} f$ will be conserved, where $A$ is the ion amplitude and $f$ is the axial frequency. When the trap becomes sufficiently shallow, ions escape. Of course, the actual escape of ions is irreversible (hence nonadiabatic); therefore, the adiabatic invariant cannot be used to calculate the energy distribution of ions in the trap. However, for most ions which remain in the trap the change in potential is adiabatic. Since the time of the predump is short compared to the ion-ion interaction time, the predump represents a reversible process for ions remaining in the trap.

A further refinement used in some measurements was the addition of a "precool" sequence. Since higher amplitude ions of a given charge state require higher voltage to 


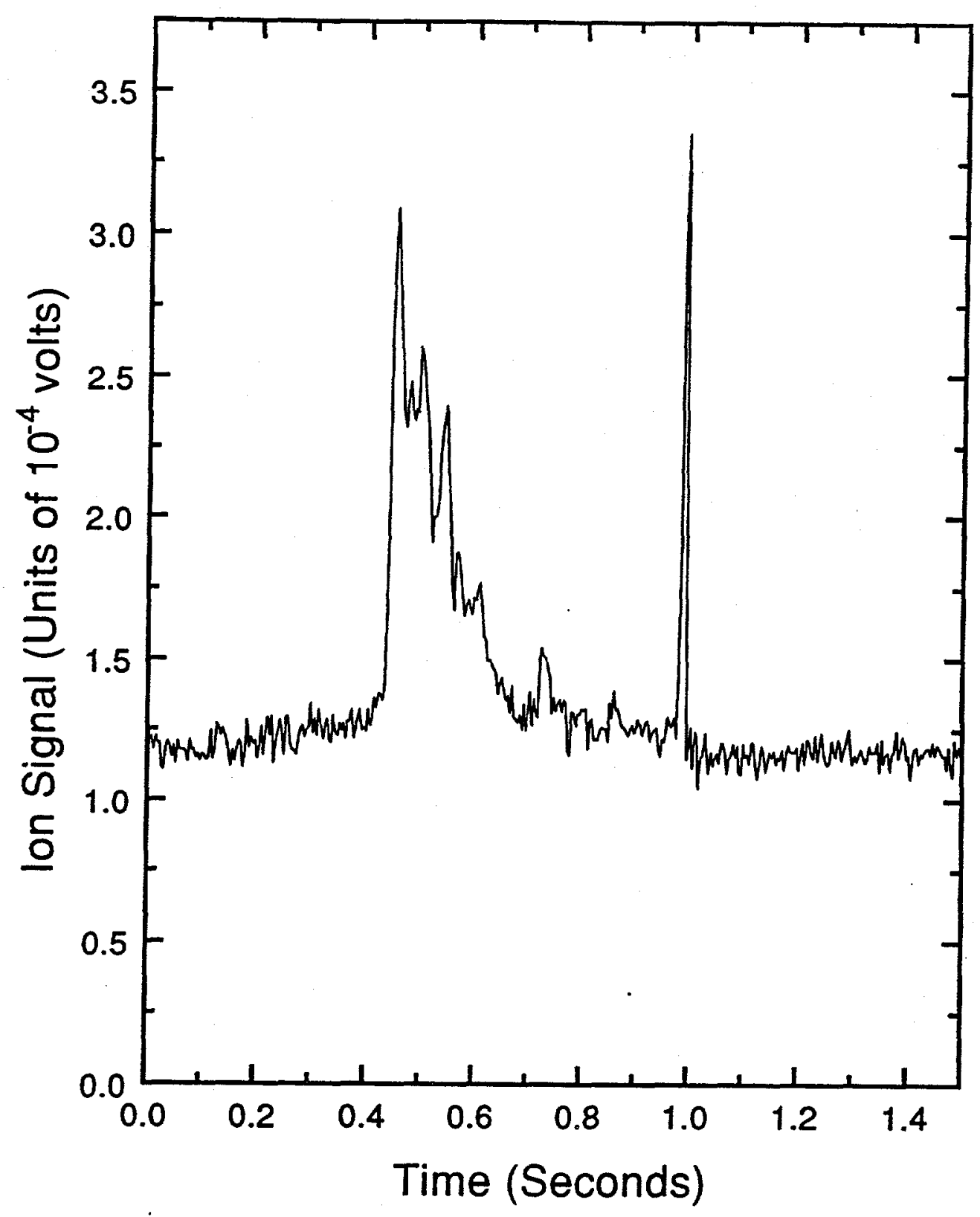

Figure 4.7: Injected ion signal average without predump. The signal is the average of about 30 cycles and represents several hundred ions. The broad peak ( $0.3-0.6$ seconds) is due to the endcap voltage ramping up through resonance, the narrow peak ( 1 second) is due to the endcap voltage quickly ramping down again. The first peak is broad because the potential in the trap is not a perfect quadratic, and it requires a higher voltage to bring higher amplitude ions onto resonace. The oscillations in the broad peak are assumed to be due to statistics. The (roughly) constant background level is due to thermal noise across the tuned circuit. 


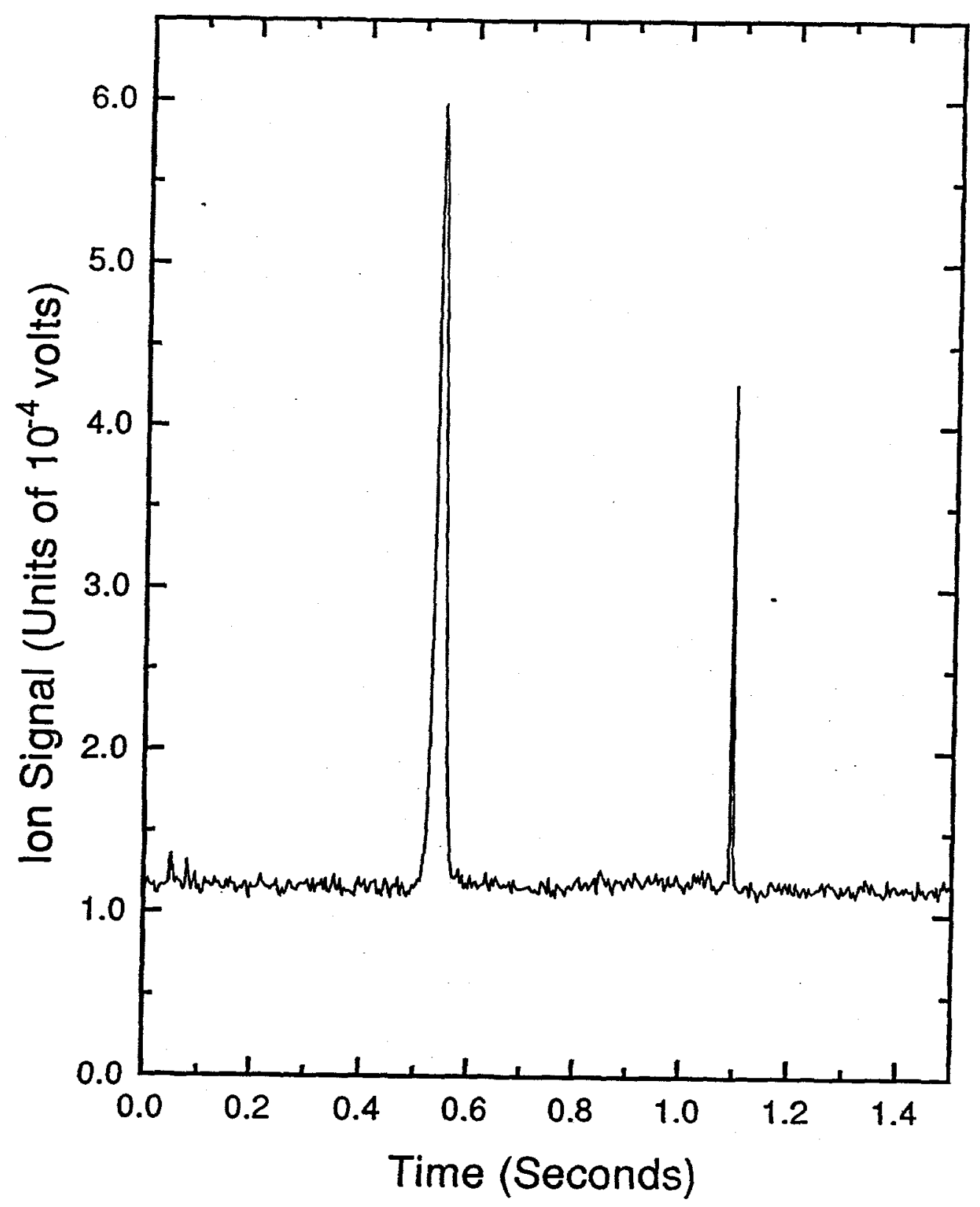

Figure 4.8: Injected ion signal average with predump. The peak has narrowed considerably after ejecting the higher amplitude ions. 
bring them onto resonance, by raising the endcap voltage to a slightly higher voltage than required to bring low amplitude ions onto resonance, the higher amplitude ions (and only the higher amplitude ions) were cooled. As the voltage on the endcaps was slowly lowered, the higher amplitude ions continued to cool (and remain on resonance), until the voltage reached the resonance voltage for low amplitude ions. A diagram of the predump-precool sequence with typical values for voltage and time is shown in Figure 4.9 .

Because of the very strong frequency dependence of the cooling rate, the energy of the initially low energy ions remained nearly unchanged. The effect of precooling is to allow a more shallow predump; thus more ions per cycle could be studied. A disadvantage is that charge transfer can occur during the precool, thus decreasing the number of ions of the injected charge state at the time of the first measurement. For this reason, precooling was not used when charge transfer times were short (no precool was used in the thorium measurements).

By observing the signal during a single injection cycle, it was possible to measure the signal due to a single ion. The peak due to a single ion is about one third the width of the averaged peak in Figure 4.8, thus even with a predump the width of the averaged signal peak is primarily determined by frequency homogeneity. The single ion peak width is determined by the quality factor of the tuned circuit:

As time progresses, the injected ions decay into successively lower charge states. Figure 4.10 shows the averaged spectrum analyzer signal after 80 seconds, in which peaks due to five charge states are clearly visible. Note that predumping is necessary to resolve the different charge states. Integrating under the peaks allows the calculation of the relative number of ions in each charge state.

In the initial RETRAP experiments [23] each captured pulse of ions was brought onto resonance only once, then dumped. Computer control of the spectrum analyzer made it possible to bring the same ions onto resonance at a fixed time interval up to 20 times. This limit (20 measurements per cycle) was set by computer memory considerations. The current technique not only vastly decreased the time required to 


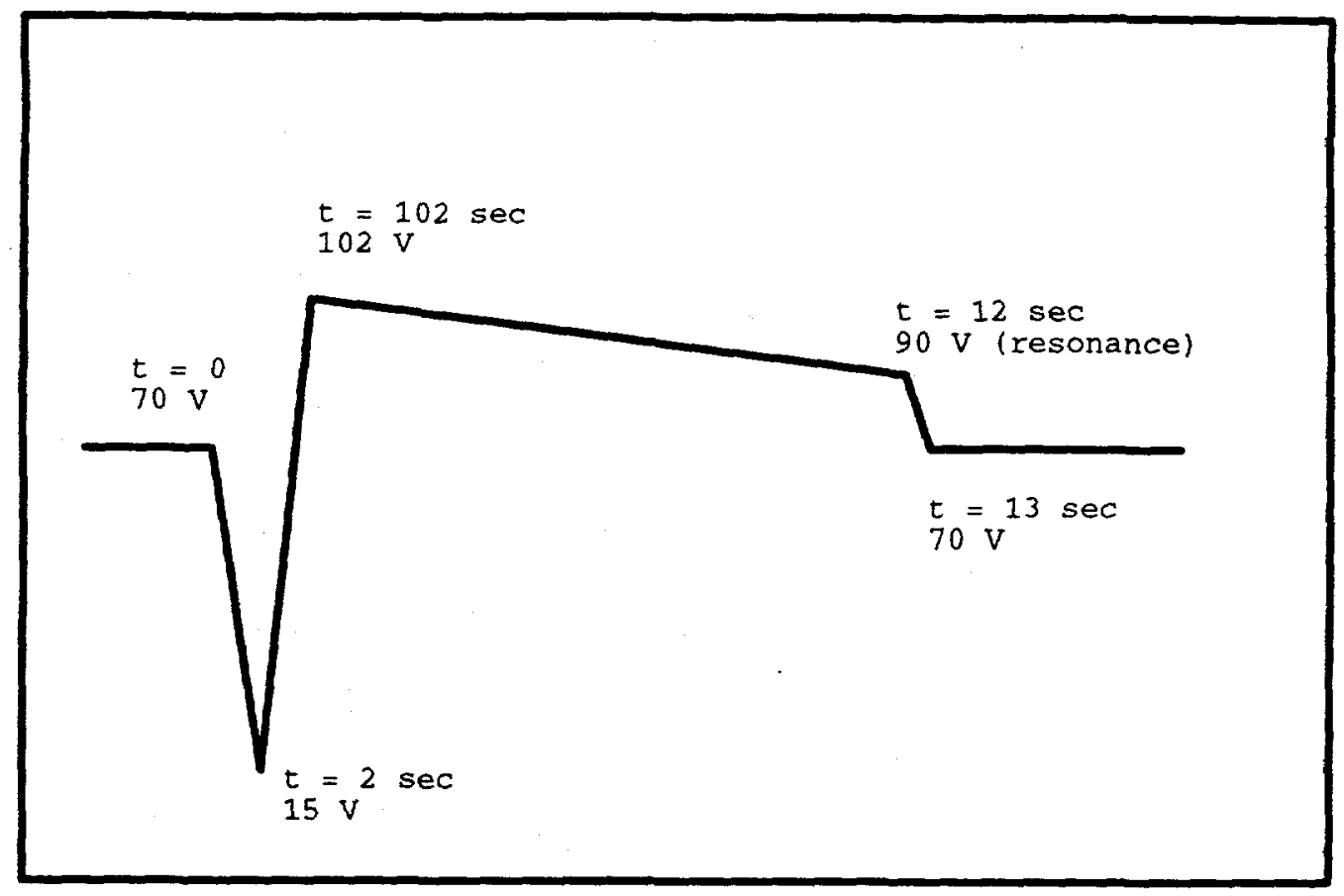

Figure 4.9: Endcap electrode voltages during predump-precool. 
take data, it made the experiment relatively insensitive to variations in the number of ions emitted by EBIT. A schematic of the charge exchange measurement cycle is shown in Figure 4.11. 


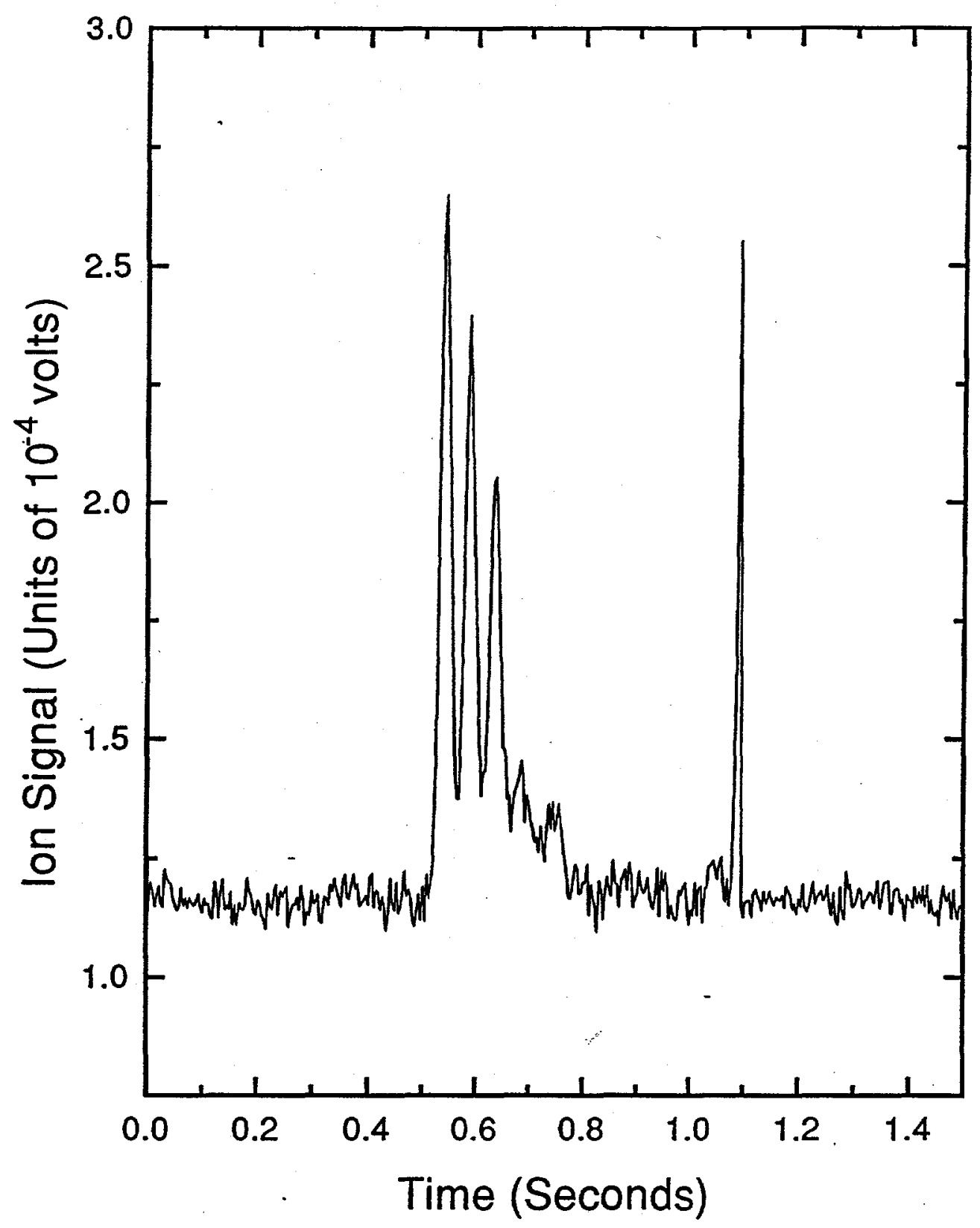

Figure 4.10: Ion signal average after 80 seconds storage time. The different peaks represent different charge states. 


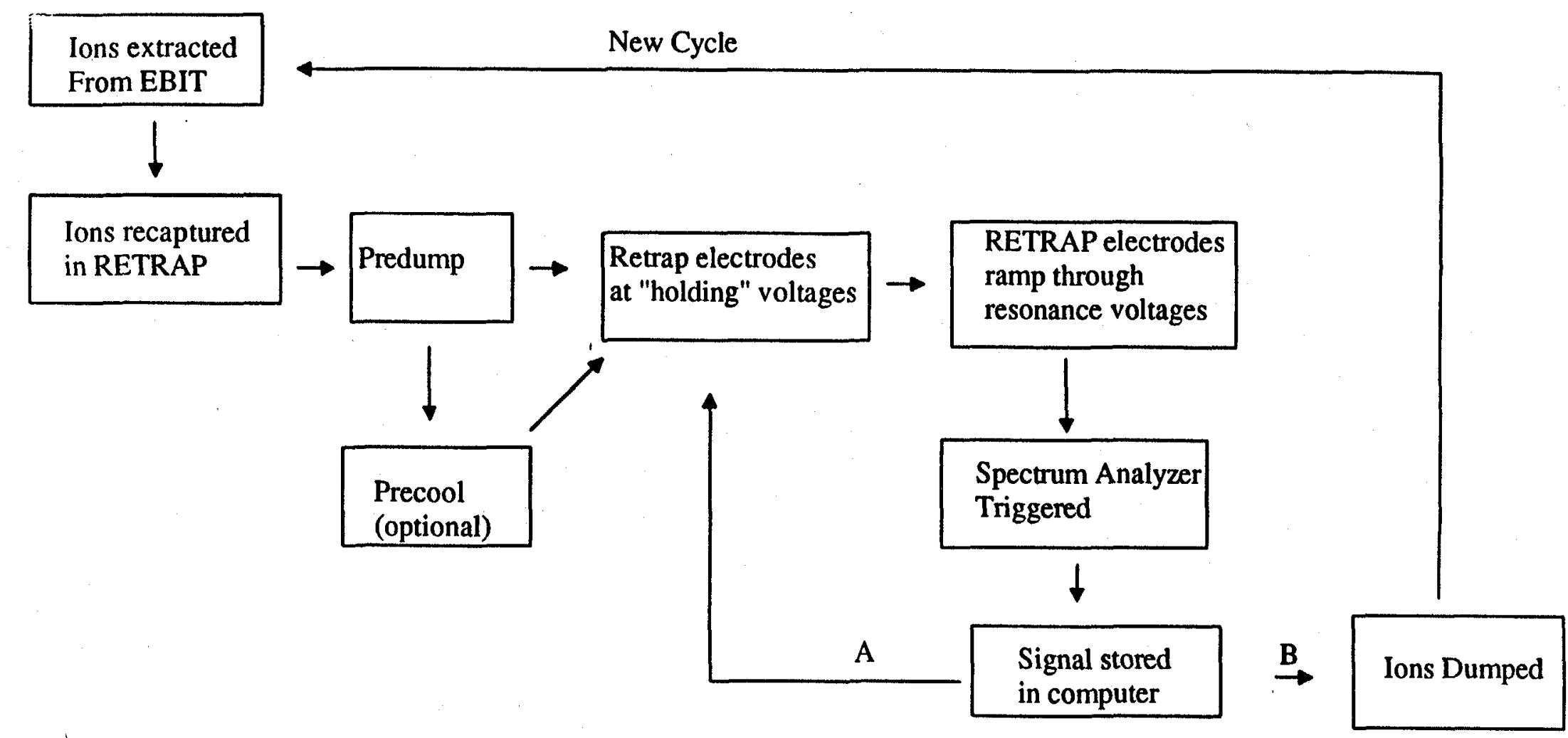

Figure 4.11: Trapping cycle schematic. Usually the same ions were brought onto resonance 20 times per cycle. The delay between successive sweeps of the same cycle was typically 5-10 seconds. 


\section{CHAPTER V}

\section{ANALYSIS}

\section{A. Introduction}

The ion signal vs. time data was analyzed in two ways: "analog" mode, in which the ion signal at regular intervals after injection was averaged over many cycles, and "digital" mode, in which each scan is studied individually in order to see individual ions changing charge.

\section{B. Digital Mode}

"Digital" mode is conceptually simpler; one observes the ions from sweep to sweep and merely counts how many ions are in each charge state at each time, based on the known position of the peak due to each charge state. Since the positions of the peaks due to various charge states are known, it is possible to determine the number of electrons an ion gains between sweeps. It is impossible to distinguish a double electron capture from successive single electron captures in the case of any particular ion, although statistics on how often an ion gains zero, one, two, or more electrons can be used to calculate a ratio of single to double electron capture rates.

For example, Figure 5.1 shows three sweeps of the same cycle. At $t_{1}$ there is only one peak, at a position indicating $\mathrm{Th}^{79+}$. At $t_{2}$ the peak is smaller, and a new peak has appeared at the $\mathrm{Th}^{77}$ position. At $\mathrm{t}_{3}$ the $\mathrm{Th}^{79+}$ peak is unchanged, the $\mathrm{Th}^{77+}$ peak is gone, and a new peak has appeared at the $\mathrm{Th}^{76+}$ position. After observing the signal through 20 cycles, it can be concluded that the peak in the first sweep represents 2 ions and in the later sweeps each peak represents 1 ion. Although it is possible that two ions will happen to be in the same charge state during every sweep of a cycle (thus, they will 
79787776

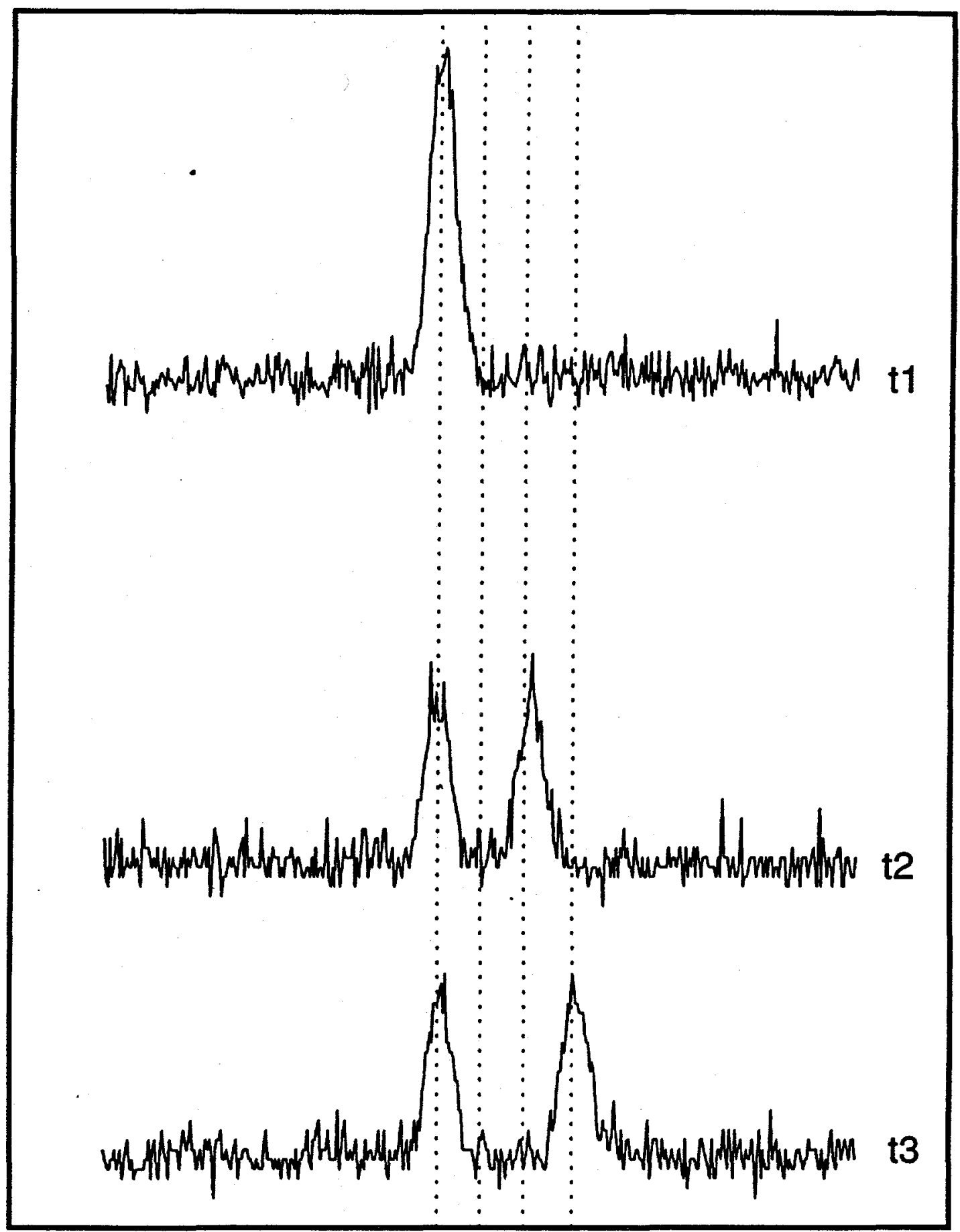

Figure.5.1: Individual ion signals. The spectrum analyzer output for three successive sweeps is shown. 
be counted as one ion) this is statistically rare and also should not bias the calculated charge transfer time one way or the other.

The main advantage of "digital" mode is that it avoids complications caused by changes in ion energy (discussed under analog mode). If a peak is known to represent a single ion, one can ignore any changes in the area of that peak, concentrating only on changes in its position. Also, one can verify that ion loss from a given charge state is indeed due to charge exchange. If, for example, ions were to somehow escape the trap axially or radially, peaks would seem to simply disappear from one sweep to the next rather than changing size or position. Absence of such disappearances indicates that all ion loss is indeed due to charge exchange. Digital mode analysis is therefore more reliable in the cases in which it can be applied.

Digital mode analysis can also be used in some cases where analog can not. When the ions have a sufficiently large axial amplitude it becomes impossible to resolve the separate charge states. However, since the position of the peak due to an ion moves in discrete steps, it is possible to determine whether an ion undergoes charge exchange (and how many times) even when it is unknown what charge state the ion started in. Since for high $\mathrm{q}$ the charge exchange rate varies only slightly from one charge state to the next, a charge exchange rate can be calculated.

However, digital mode can only be used effectively when the number of ions captured is few enough that ions can be observed to decay individually (if more than about 3 ions are captured in a single cycle, it may become impossible to say with confidence how many ions a peak represents). This has not generally been a limiting factor in RETRAP experiments so far, (too few ions are more often the problem than too many) although it may be in future experiments.

Another limitation on the use of this technique is that the signal to noise ratio of the signal from the preamplifier is such that single ion peaks cannot be distinguished for ions with $q$ less than $\sim 30$ at the energies of these experiments. This means that the lifetime measurements of low charge state ions had to be done in analog mode (see 
pressure calibration), and in future experiments done at lower energies it may be impossible to resolve single ion peaks even for the highest charge states of ions.

C Analog mode

As was discussed above, when there are many ions in the trap or their charge state or energy is too low, it is impossible to observe the charge transitions of individual ions and some form of averaging must be used. When analyzing ions captured from EBIT this is an average over many trapping cycles, although when injecting from the MeVVA sometimes only a single cycle is used because the number of ions is large (about $10^{5}$ ) and the cycle time is relatively long. The method of analyzing these data is referred to as analog mode because it is a signal strength rather than a number of discrete ions which is measured. Analog mode analysis thus gives the relative populations of the various charge states rather than absolute numbers.

The average voltage squared on the preamplifier due to $\mathrm{N}$ ions oscillating incoherently is given by

$V^{2}=N R^{2} q^{2} \gamma^{2} \omega_{z}^{2} A^{2} / 4 d^{2}$

where $\mathrm{R}=\mathrm{Q} / \omega \mathrm{C}$ is the effective parallel resistance of the tuned circuit at resonance, $\mathrm{A}$ is the average oscillation amplitude, and $\gamma$ is a coupling factor with a value of about 0.9 [21]. The total voltage at the preamplifier $\mathrm{Vp}$ also has a component $\mathrm{V}_{\mathrm{T}}$ due to the thermal Johnson noise across the input resistor. The actual ion signal is thus related to the measured signal by

$\mathrm{S}=\mathrm{V}_{\mathrm{P}}^{2}-\mathrm{V}_{\mathrm{T}}^{2}$ 
Figure 5.2 shows the integral of $S$ for the various peaks at time intervals of 10 seconds, averaged for 38 injection cycles. . The value for $V_{T}$ used was the averaged spectrum analyzer signal with no ions present in the trap.

It is evident from eqn. 5.1 that for ions of equal amplitude, $\mathrm{S}$ is proportional to $q^{2}$

The axial energy of an ion is given by

$U=m \omega^{2} A^{2} / 2$

As the voltage on the endcaps increases, the ions are heated. Since the process is adiabatic, the quantity $J_{Z}=\omega A^{2}$ is a constant for each ion. Recall from equation 4.15 that $\omega$ is proportional to $\sqrt{q V}$. Thus, the "holding" amplitude $\mathrm{A}_{\mathrm{H}}$ is related to the resonance amplitude $A_{R}$ by

$A_{R}^{2}=A_{H}^{2}\left(V_{H} / V_{R}\right)^{1 / 2}$

where $V_{H}$ is the endcap voltage during the holding phase and $V_{R}$ is the voltage when the ions are on resonance. Assuming ions of each charge state have the same kinetic energy while held off resonance, the holding amplitude $A_{H I}$ of an ion with charge $q_{1}$ is related to the amplitude of an ion with charge $q_{2}$ by

$\mathrm{A}_{\mathrm{H} 1}{ }^{2} \mathrm{q}_{1}=\mathrm{A}_{\mathrm{H} 2}{ }^{2} \mathrm{q}_{2}$

The voltage required to bring an ion onto resonance varies inversely with $q$,

$\mathrm{V}_{\mathrm{R} 1} \mathrm{q}_{1}=\mathrm{V}_{\mathrm{R} 2} \mathrm{q}_{2}$

Thus, for ions of equal initial kinetic energy, 


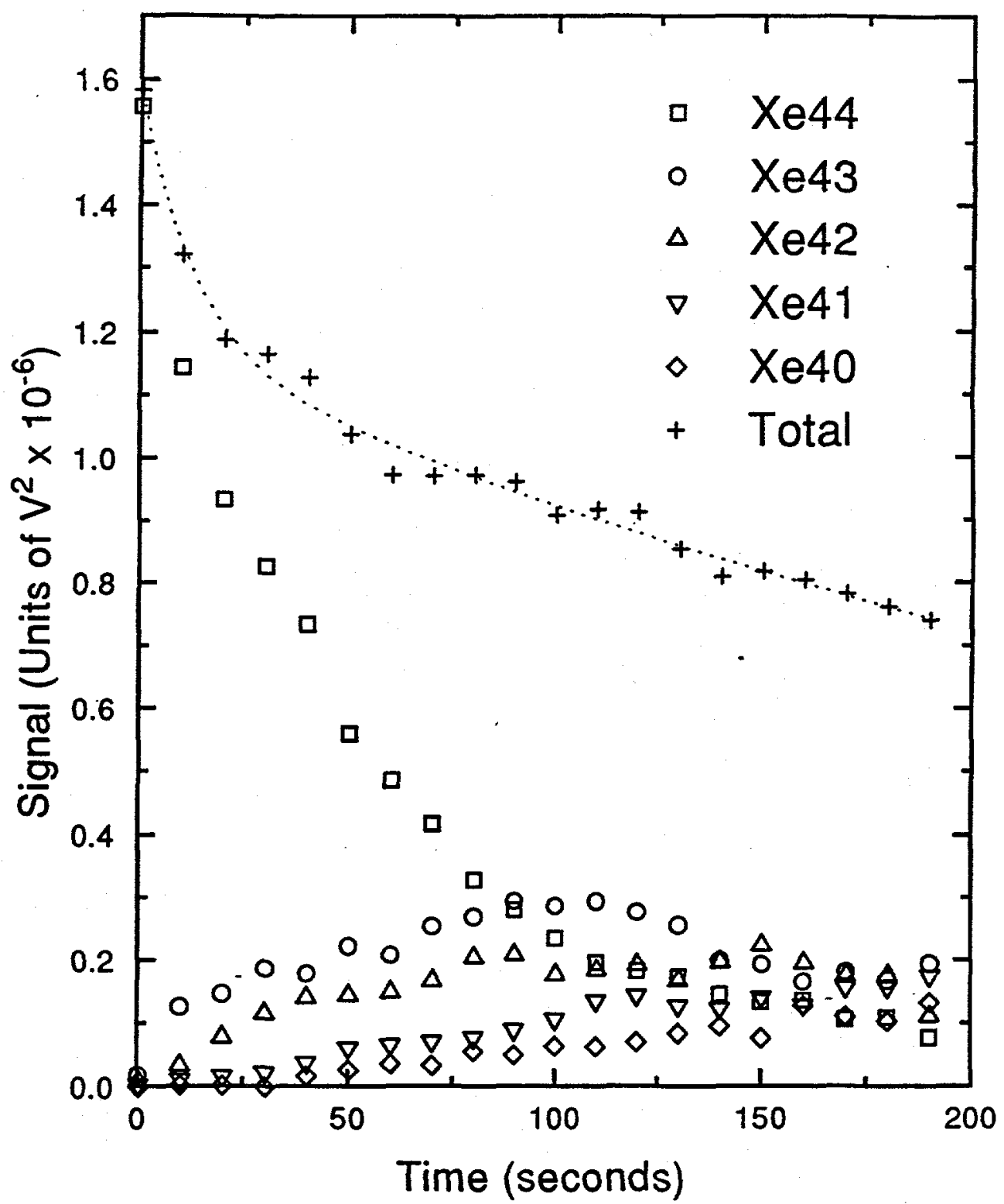

Figure 5.2 Averaged Ion Signals

Figure 5.2: Analog mode ion signal data. The "total" curve has been fitted to the equation Total $=A^{*}\left(\left(1+B^{*} \exp \left(-t / t_{1}\right)\right)^{*} \exp \left(-t / t_{2}\right)\right.$. 
$A_{R 1}^{2}=A_{R 2}^{2}\left(q_{2} / q_{1}\right)^{1 / 2}$

so $S$ is proportional to $\mathrm{q}^{3 / 2}$.

The "total" curve is the summed $S$ for all charge states present in the trap, adjusted for the signal strength of the charge state. The decrease in the "total" curve was evidently due to either a decrease in ion amplitude or an ion loss mechanism other than charge exchange.

The width of the spectrum analyzer signal peak for the injected ions was used to estimate their energy, based on the frequency dependence of ion amplitude (see Figure 4.5). Their maximum energy was about 16 volts times the charge. The average kinetic energy is assumed to be about $1 / 4$ of this (one factor of two because the average energy is probably about $1 / 2$ the maximum energy, another factor of two because averaged over time $1 / 2$ the energy is potential and $1 / 2$ is kinetic), giving an average kinetic energy of $176 \mathrm{eV}$ in the lab frame for $\mathrm{Xe}^{44}$. In the center of mass frame for a xenon-hydrogen collision, this is $2.6 \mathrm{eV}$. The center of mass energy for thorium-hydrogen collisions is nearly the same, since (for the particular ions studied) the charge to mass ratio for thorium and xenon are nearly the same.

Ions can eventually diffuse radially out to the walls of the trap due to ion-ion collisions or collision with neutrals which do not result in charge exchange. The ions are thus eventually lost. However, the time required for this is apparently quite large compared to charge exchange lifetimes for highly charged ions. For example, $\mathrm{Be}^{2+}$ was injected from the MeVVA atop RETRAP for calibration purposes. The decay time for the $\mathrm{Be}^{2+}$ signal was over 4000 seconds. This decay time includes contributions from electron capture and diffusion, and must thus be faster than either individually. Ion diffusion time is proportional to $\omega_{c}^{-2}[1]$ so it should occur on a similar time scale for $\mathrm{Be}^{2+}$ and highly charged ions. The lifetime of $\mathrm{Be}^{2+}$ was measured for magnetic fields ranging from 2 Tesla to 4 Tesla, and the measured lifetime did not appreciably change. It is thus concluded that radial diffusion is a negligible effect even on a 4000 second time scale, and certainly on the time scale associated with decays of highly charged ions. 
Next we consider a loss in axial signal amplitude due to ion-ion collisions.

Although total energy is conserved, if the ions are injected with more axial energy than transverse energy, ion-ion collisions will transfer axial energy into transverse energy, resulting in a signal drop. The rate $v$ for such collisions (see [24] and [25]) is given by

$v=\left(8 \pi^{1 / 2} / 15\right) n b^{2} v \ln [\Lambda]$

where $n$ is the ion density, $b=q^{2} / 4 \pi \varepsilon_{0} E$ is the classical distance of closest approach, $v$ is the average ion velocity, and $\Lambda=r_{c} / b$ where $r_{c}$ is the cyclotron radius and $E$ is the ion energy. Note that, holding energy constant, the collision rate is proportional to $\mathrm{q}^{4}$, while holding amplitude constant the collision rate is proportional to $\mathrm{q}^{2}$. For $\mathrm{Xe}^{44+}$ with maximum kinetic energy 10 volts times the charge, $b$ is $6.3 \times 10^{-9}$ meters and $v$ is 1.75 $\times 10^{4}$ meters/second. For an energy in cyclotron motion of $2 q$ volts, $r_{c}$ is $3.4 \times 10^{-4}$ meters so $\Lambda$ is about 10. Density is estimated to be $4 \times 10^{8} / \mathrm{m}^{3}$, so the estimated rate is $v=2.8 \times 10^{-3} \mathrm{sec}^{-1}$. The differential equation for axial energy is

$\mathrm{dT}_{\mathrm{ax}} / \mathrm{dt}=-2 v\left(\mathrm{~T}_{\mathrm{ax}}-\mathrm{T}_{\mathrm{tr}}\right)$

where $T_{a x}$ is the axial temperature, $T_{t r}$ is the transverse temperature, and the average temperature (which is constant in time) is

$T_{a v}=\left(2 T_{t r}+T_{a x}\right) / 3$

The factor of 2 comes in because there are two transverse degrees of freedom. Solving eqn (5.9) yields

$T_{a x}(t)=A^{*} \exp (-3 v t)+T_{a v}$ 
giving a time constant for equilibration of energy of about 120 seconds. It should be noted that this is a very rough estimate, since the density of ions in the trap is not well known and will not be constant between experiments. The important result is that energy equilibration may take place on about the same time scale as charge exchange, even with only a few ions in the trap.

There is some cooling each time the ions pass through resonance during a ramp for detection purposes. If the time of the ramp is $t_{r}$, the voltage of the ramp is $V_{r}$, each time the ions sweep through resonance, they cool as if they spend an effective time $t_{\text {eff }}$ on resonance, where $t_{\text {eff }}$ is approximately given by

$$
\mathrm{t}_{\mathrm{eff}}=\mathrm{t}_{\mathrm{r}}\left(2 \mathrm{~V}_{0} /\left(\mathrm{V}_{\mathrm{r}} * \mathrm{Q}\right)\right)
$$

In these measurements $t_{r}$ was about 2 seconds and $V_{r}$ was about 40 volts. This corresponds to about .05 seconds on resonance each sweep, or 1 second after 20 sweeps. Since the one dimensional cooling time is about 1 second, this effect is too small to explain all the decrease of "total" signal, but may not be negligible. In order to experimentally measure this effect, a Xe ${ }^{44+}$ charge exchange time measurement was repeated, changing the time between sweeps from 10 seconds to 20 seconds. As expected, the total signal decreased faster at 10 seconds between scans. The fit parameter $\mathrm{t} 2$ (see below) was about twice as high at 20 seconds between scans as at 10 .

There is also a small amount of resistive cooling while the ions are held off resonance. However, the cooling rate is about 1000 times slower at the holding voltage than at the resonance voltage, so this effect is negligible.

To compensate for these effects, the "total" curve $\Sigma(t)$ was fitted to the equation

$\Sigma(t)=A(1+B \exp (-t / t 1)) \exp (-t / t 2)$

where $A(1+B)$ is essentially the incident ion signal at $t=0, B$ gives the fraction of incident energy which is axial ( $\mathrm{B}=2$ for initially all axial, $\mathrm{B}=-1$ for initially all radial), 
$\mathrm{t} 1$ gives the time scale for equilibration of axial and angular energy, and $\mathrm{t} 2$ gives the time scale for cooling. The data points for each charge state $S_{n}$ were then rescaled by the equation

$S_{n}^{\prime}(t)=S_{n}(t) \Sigma(0) / \Sigma(t)$

Figure 5.3 shows the rescaled Figure 5.2. These rescaled data were fitted to the rate equations (see next section). Where possible data was fitted using both analog and digital methods. The results of the two methods are consistent within $10 \%$.

It should be mentioned that if an ion captures too many electrons it will no longer appear on the spectrum analyzer signal (since the endcap voltage will not go high enough to bring it on resonance), but it would be wrong to "correct" the total for its absence. However, since at least 5 charge states were summed for the total, very few ions will have "disappeared" this way before nearly all ions of the incident charge state were gone.

D. Rate Equations

Let the number of the ions in the highest charge state in the trap (the injected charge state) be $N_{1}$, the next lower charge state be $N_{2}$, etc. Let the single electron capture rate for the highest charge state be $\lambda_{11}$, the double electron capture rate be $\lambda_{12}$, the single electron capture rate for the next highest charge state be $\lambda_{21}$, etc. Finally, let $\lambda_{11}$ $+\lambda_{12}=\lambda_{1}$, etc. Then the differential equations for the densities of charge states versus time are

$\mathrm{dN}_{1} / \mathrm{dt}=-\lambda_{1} \mathrm{~N}_{1}$

$\mathrm{dN}_{2} / \mathrm{dt}=\lambda_{11} \mathrm{~N}_{1}-\lambda_{2} \mathrm{~N}_{2}$

$\mathrm{dN}_{3} / \mathrm{dt}=\lambda_{12} \mathrm{~N}_{1}+\lambda_{21} \mathrm{~N}_{2}-\lambda_{3} \mathrm{~N}_{3}$ 


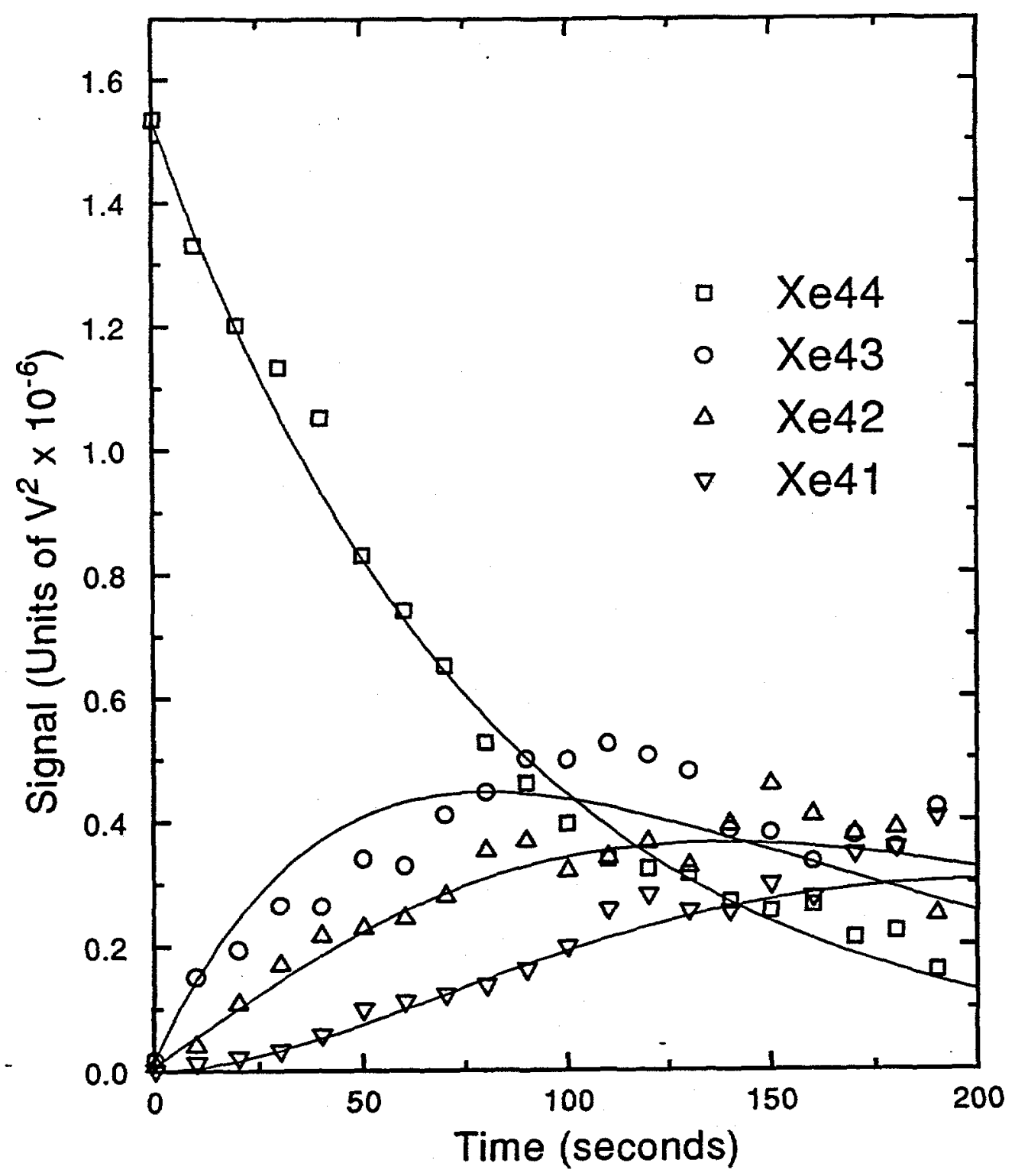

Figure 5.3: Corrected analog mode ion signal data. Data has been rescaled to compensate for the loss in total ion signal and for the difference in signal strength due to charge state. The solid lines indicate a visual fit of the rate equations to the data. For this figure double electron capture cross section is assumed to be $23 \%$ of the total. 
$\mathrm{dN}_{4} / \mathrm{dt}=\lambda_{22} \mathrm{~N} 2+\lambda_{31} \mathrm{~N}_{3}-\lambda_{4} \mathrm{~N}_{4}$

etc.

At time $t=0$, the density of the highest charge state is $N_{10}$ and the densities of all other charge states are zero. The solutions are

$N_{1}(t)=N_{10} \exp \left[-\lambda_{1} t\right]$

$N_{2}(t)=N_{10}\left(\lambda_{11} /\left(\lambda_{2}-\lambda_{1}\right)\right)\left(\exp \left[-\lambda_{1} t\right]-\exp \left[-\lambda_{2} t\right]\right)$

$N_{3}(t)=N_{10}\left(\left(\left(\lambda_{12} /\left(\lambda_{3}-\lambda_{1}\right)\right)+\lambda_{21} \lambda_{11} /\left(\left(\lambda_{2}-\lambda_{1}\right)\left(\lambda_{3}-\lambda_{1}\right)\right)\right) \exp \left[-\lambda_{1} t\right]\right.$

$-\left(\lambda_{21} \lambda_{11} /\left(\left(\lambda_{2}-\lambda_{1}\right)\left(\lambda_{3}-\lambda_{2}\right)\right)\right) \exp \left[-\lambda_{2} t\right]$

$\left.+\left(\lambda_{21} \lambda_{11} /\left(\left(\lambda_{3}-\lambda_{1}\right)\left(\lambda_{3}-\lambda_{2}\right)\right)-\lambda_{12} /\left(\lambda_{3}-\lambda_{1}\right)\right) \exp \left[-\lambda_{3} t\right]\right)$

These equations may be modified to allow for an initial population of lower charge states in a rather straightforward way i.e. if we introduce $N_{20}=N_{2}(t=0)$

$N_{1}{ }^{\prime}(t)=N_{1}(t)$

$N_{2}^{\prime}(t)=N_{2}(t)+N_{20} \exp \left[-\lambda_{2} t\right]$

$N_{3}{ }^{\prime}(t)=N_{3}(t)+N_{20}\left(\lambda_{21} /\left(\lambda_{3}-\lambda_{2}\right)\right)\left(\exp \left[-\lambda_{2} t\right]-\exp \left[-\lambda_{3} t\right]\right)$

These equations rapidly increase in complexity of coefficients for $\mathrm{N}$ greater than 3 .

However, given the charge exchange coefficients and the initial populations, the 
expected population of any charge state may be calculated iteratively from the differential equations.

The rate equations also give the probabilities $P_{n}$ of an ion capturing $n=$ zero, one, or two electrons in a given time interval (by setting $N_{10}=1$ ). This is useful in evaluating the fraction of double capture. Since (as it turns out) the ratio of double to . single electron capture is rather small $(\sim 20 \%)$, double capture has a relatively small impact on the population of charge states vs. time. However, since $\lambda_{1} t$ is also small (typically .3) between two measurements, double capture is responsible for a significant fraction ( $\sim 3)$ of events in which an ion captures two electrons between measurements.

In evaluating the fraction of double capture by this method, only those cycles in which exactly one ion was present were used. This is because an ambiguity can arise with multiple ions present. For example, if in one measurement there is one $\mathrm{Th}^{79+}$ ion and one $\mathrm{Th}^{78+}$ ion and in the succeeding measurement there is one $\mathrm{Th}^{78+}$ ion and one $\mathrm{Th}^{77+}$ ion, the $\mathrm{Th}^{79+}$ may have gained two electrons or each ion could have gained one electron. Although in many cycles there will be no such ambiguity, rejecting some cycles as ambiguous while retaining others would probably bias the data. Another example: again initially there is one $\mathrm{Th}^{79+}$ and one $\mathrm{Th}^{78+}$ ion. If, at a later time, there are two $\mathrm{Th}^{78+}$ ions this is recorded as a single capture whereas if at a later time there is one $\mathrm{Th}^{78+}$ and one $\mathrm{Th}^{77+}$ the cycle is rejected as ambiguous. Clearly this would bias results against double capture.

Since there were few cycles with exactly one ion, the same ion was observed through several changes of charge in order to improve statistics. The charge exchange cross sections of adjacent charge states (for both single and double capture) were assumed to be equal. Under these assumptions the probabilities of an ion capturing $n=$ 0,1 , and 2 electrons are

$$
\begin{aligned}
& P_{0}(t)=\exp \left(-\lambda_{T} t\right) \\
& P_{1}(t)=\lambda_{s} t \exp \left(-\lambda_{T} t\right)
\end{aligned}
$$


$P_{2}(t)=\left(\lambda_{D} t+\lambda_{s}^{2} t^{2} / 2\right) \exp \left(-\lambda_{T} t\right)$

where $\lambda_{s}$ is the single electron capture rate, $\lambda_{D}$ is the double electron capture rate, and $\lambda_{T}=\lambda_{S}+\lambda_{D}$ is the total charge change rate.

\section{E. Neutral Density Calibration}

Pressure at the outside of the RETRAP chamber was measured via an ionization gauge. Typical readings were about $2 \times 10^{-10}$ torr with the helium dewar filled. The pressure is much lower inside the actual trap, because the helium dewar acts as a cryopump. Because the ionization gauge reading may contain contributions from gasses which will completely freeze out at liquid helium temperatures, it may not even be proportional to pressure inside the trap.

It was decided that the best way to measure the neutral density in the trap was by measuring the reaction rate of an ion with known charge exchange cross section. The density is then given by

$\mathrm{n}=\mathrm{k} / \mathrm{ov}$.

Two ions were chosen for this purpose: $\mathrm{Ar}^{11+}$ and $\mathrm{Be}^{2+}$.

$\mathrm{Ar}^{11+}$ was chosen because a preprint (now published [3]) had been made available to the RETRAP group giving cross section dependence on energy over a wide range, which included the energy range of stored ions in RETRAP. Also, the charge to mass ratio is fairly near that of the highly charged ions of interest in RETRAP ( $\mathrm{m} / \mathrm{q}$ is 3.63 for $\mathrm{Ar}^{11+}, 3.09$ for $\mathrm{Xe}^{44+}, 3.22$ for $\mathrm{Th}^{72+}$, and 2.93 for $\mathrm{Th}^{79+}$ ).

Measurements of the charge transfer rates yields the product nv rather than giving the density directly. Velocity can be estimated based on the width of averaged ion signal peaks and the known anharmonicity of the trap, but this gives only an estimate 
(primarily an upper bound on ion energy) because it is impossible to distinguish broadening due to axial amplitude from broadening due to radial position. Therefore these numbers are only used in establishing the energy region of applicability of these results.

The relative velocities of $\mathrm{Ar}^{11+}$ and the highly charged ions in RETRAP are better known than the absolute velocities. Since the $\mathrm{m} / \mathrm{q}$ ratios vary by less than $20 \%$ for the ions above, their velocities at the same amplitude in the same trap depth would vary by only $10 \%$. However, the voltage on the endcaps was kept higher for states with higher $\mathrm{m} / \mathrm{q}$ ratios since they must all be brought to the same resonant frequency. Two ions with the same frequency and the same amplitude must also have the same velocity. The velocities of the highly charged ions and the $\mathrm{Ar}^{11+}$ are thus assumed to be the same within $20 \%$.

Studies of individual trapping cycles revealed that, in a few cycles, ions of a higher charge state were captured along with the $\mathrm{Ar}^{11+}$. These ions could clearly be identified as a contaminant because their signals were much bigger than the $\mathrm{Ar}^{11+}$ signals, and because the distance their signals moved was much smaller than that for $\mathrm{Ar}^{1+}$, indicating a smaller relative shift in $\mathrm{q} / \mathrm{m}$ and hence a higher initial $\mathrm{q}$. These ions must have had nearly the same $\mathrm{q} / \mathrm{m}$ as $\mathrm{Ar}^{11+}$ in order to be bent the proper angle by the charge selecting magnet. Based on the separation of the peaks of the charge states of the contaminant, and its known $\mathrm{q} / \mathrm{m}$, the contaminant was tentatively identified as $\mathrm{Ba}^{38+}$ or perhaps $\mathrm{Xe}^{37+}$. Barium is always present in EBIT in small amounts because it is sputtered from the electron gun. Xenon injected earlier may have adhered to cold surfaces in EBIT and been desorbed later. Since the contaminant was only present in a few cycles, ( 4 contaminant ions in about 100 cycles) those cycles were simply removed from the average data when calculating $\mathrm{Ar}^{11+}$ charge exchange rates. Although it is impossible to draw any strong conclusions from so few samples, the amplitudes of single ion signal peaks of the contaminants seemed to indicate about the same oscillation amplitude as for the highly charged ion measurements. 
An attempt to calibrate the neutral density using a beryllium MeVVA mounted above RETRAP was also made. Use of the MeVVA allowed measurements to be made before and after beam times, and the number of ions captured was so high that only one cycle was required to get reasonably good statistics. Beryllium was chosen because the $\mathrm{m} / \mathrm{q}$ ratio for $\mathrm{Be}^{2+}(4.5)$ is reasonably close to that of the highly charged ions, and because $\mathrm{Be}^{1+}$ has a transition that can be used for laser cooling (this will be used in future experiments).

There is no charge analyzing magnet between the MeVVA and RETRAP, so ion pulses captured from the MeVVA contain a mixture of $\mathrm{Be}^{2+}, \mathrm{Be}^{1+}$, and various contaminants including $\mathrm{H}_{2}^{+}, \mathrm{Mg}^{+}$and $\mathrm{Mg}^{2+}$. The number of these ions present is unknown (even as a relative quantity) because the maximum voltage which can safely be applied to the endcap electrodes is only sufficient to bring ions with a mass to charge ratio of about 10 onto resonance.

Figures 5.4 and 5.5 show the signal of $\mathrm{Be}^{2+}$ and $\mathrm{Be}^{1+}$ vs. time. As can be seen, the $\mathrm{Be}^{1+}$ signal continues to rise nearly linearly out to 4000 seconds, while the $\mathrm{Be}^{2+}$ signal has decreased by more than a factor of 3 . Also, the $\mathrm{Be}^{1+}$ signal rises much more slowly than would be expected from the decrease in the $\mathrm{Be}^{2+}$ signal. The exponential fit to the $\mathrm{Be}^{2+}$ gave a decay time of 3000 seconds. The first point was neglected because at very early times there is a rapid decrease in signal due to axial energy being transformed into angular energy. However, due to the large number of ions and the relatively long time scales involved, the energies of the degrees of freedom should have come into equilibrium long before the second sweep. Note also that the fit is rather poor, indicating the mechanism for the decrease of the $\mathrm{Be}^{2+}$ signal is not well described by a single exponential.

Since it is energetically unfavorable for $\mathrm{Be}^{+}$to capture electrons from $\mathrm{H}_{2}$, the relation of the number of $\mathrm{Be}^{2+}$ ions $\mathrm{N}_{2}$ to the number of $\mathrm{Be}^{+}$ions $\mathrm{N}_{1}$ is (in the absence of other loss mechanisms)

$\mathrm{dN}_{1} / \mathrm{dt}=\mathrm{N}_{2} / \tau$ 


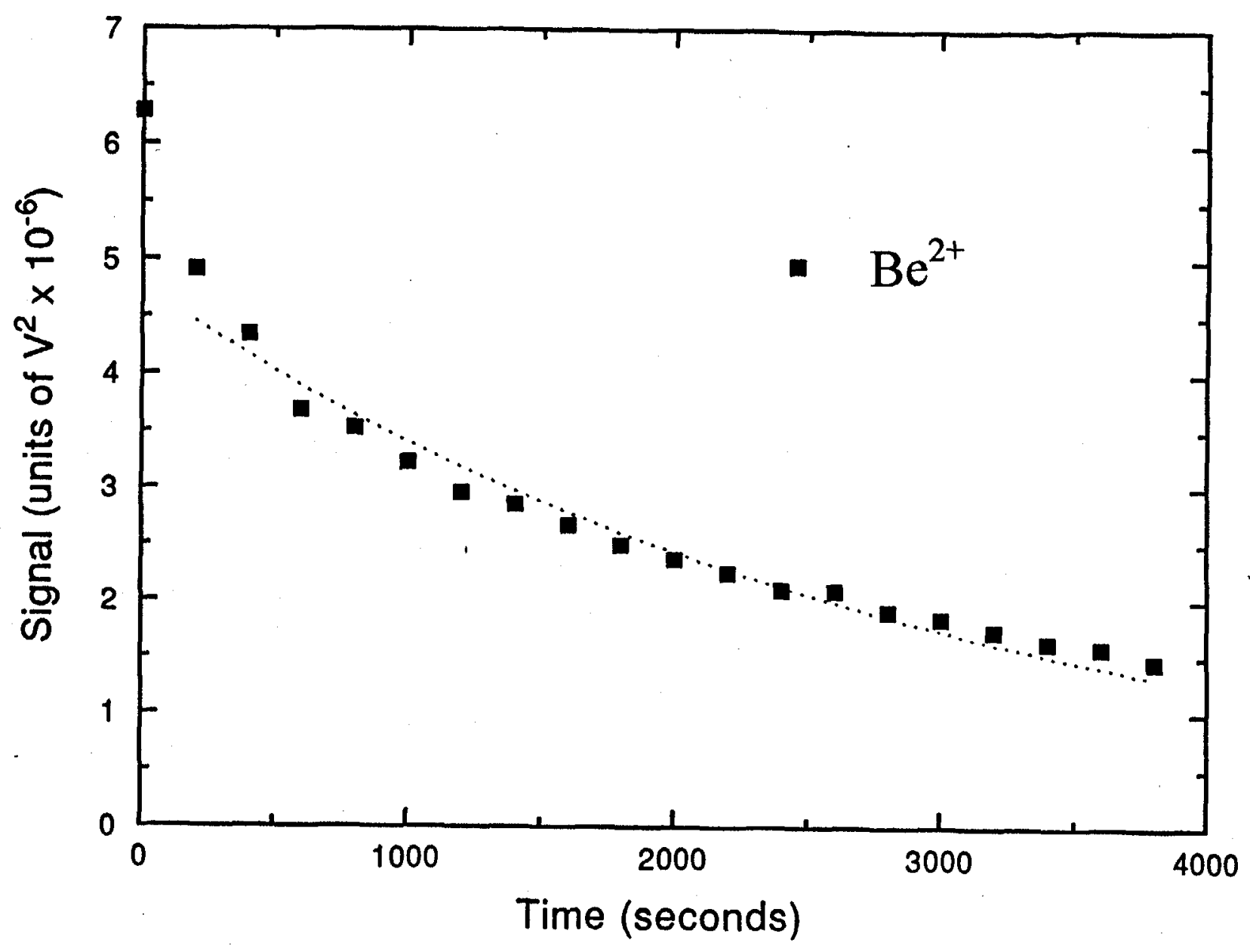

Figure $5.4: \mathrm{Be}^{2+}$ signal vs. time. The signal decreases faster at earlier times, probably indicating that the ions are cooling (and perhaps evaporating) as well as changing charge. 


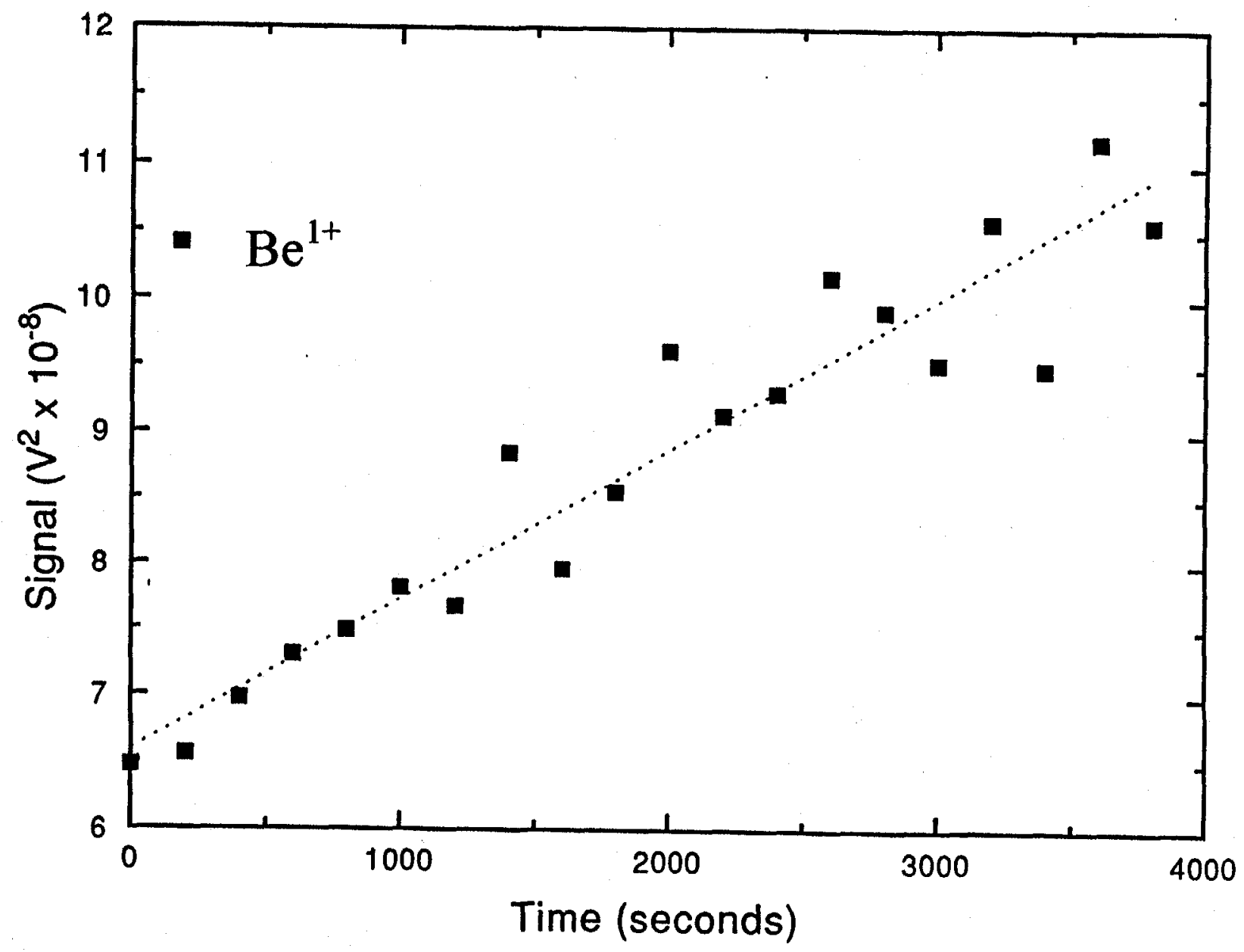

Figure 5.5: $\mathrm{Be}^{1+}$ signal vs. time. 
where $\tau$ is the charge exchange lifetime of the $\mathrm{Be}^{2+}$. Scaling $\mathrm{Be}^{1+}$ signals for charge, this gives a charge exchange lifetime of $5 \times 10^{4}$ to $1.5 \times 10^{5}$ seconds depending on at what time the equation is evaluated. Cooling may explain some of this discrepancy; however, as can be seen from equation 4.20 the $\mathrm{Be}^{2+}$ cools about 20 times slower than $\mathrm{Xe}^{44+}$ on resonance. Since the $\mathrm{Be}^{2+}$ is only near resonance for a short time each measurement, there must be some other loss mechanism.

In principle, when ions exchange axial energy in collisions one might gain enough energy to escape the trap axially. This is nearly impossible for the highly charged ions, since the average ion kinetic energy is estimated to be about $1 / 10$ the well depth and there are generally fewer than 10 ions in the trap. However, there are many thousands of ions in the trap when injecting from the MeVVA. Since the $\mathrm{Be}^{1+}$ and $\mathrm{Be}^{2+}$ ions are in mutual thermal equilibrium, they will have on the average the same kinetic energy, but since the singly charged ions require only half as much kinetic energy to escape, virtually all ions which escape will be singly charged.

Axial escape of the $\mathrm{Be}^{+}$is currently considered the most likely explanation for the slow rise of the $\mathrm{Be}^{+}$signal. Since the ions remaining in the trap are cooled by the escape of the hot ions, the decrease in the $\mathrm{Be}^{2+}$ signal may not be an accurate reflection of the $\mathrm{Be}^{2+}$ charge exchange time. Further, the velocity of $\mathrm{Be}^{2+}$ may be substantially lower than for the highly charged ions.

Due to the difficulty in interpreting the beryllium data, it is not used as an absolute measure of neutral density in these results, and is instead used only as a demonstration that low charge state ions can exist for thousands of seconds in RETRAP. The beryllium can, however, give a maximum on the neutral density. Since the decrease in the $\mathrm{Be}^{2+}$ signal includes charge exchange and other contributions, it gives a minimum of the charge exchange lifetime. Recall that the lifetime $\tau$ is related to the neutral density $n$ by

$$
\mathrm{n}=(\tau \sigma \mathrm{v})^{-1}
$$


If the velocity is sufficiently low (so the Langevin theory applies), the reaction rate $\sigma \mathrm{v}$ is a constant. This rate has been measured, and has a value of $1.55 \times 10^{-9} \mathrm{~cm}^{3} / \mathrm{sec}( \pm 30 \%)$ [26]. At higher velocities the product $\sigma v$ increases, so assuming this minimum value for $\sigma v$ gives us a maximum for $n$. With a minimum life of 3000 seconds, this gives a maximum neutral density of $2 \times 10^{5} \mathrm{~cm}^{-3}$.

Preliminary analysis of charge transfer times indicated that neutral density increased significantly during the course of the experiment. This is understood as follows: hydrogen has a high probability of sticking to metal surfaces at $4.2 \mathrm{~K}$, however hydrogen is also desorbed from the surface at a rate proportional to the fraction of surface covered. Since hydrogen is constantly streaming in from the beamline and the warm parts of the chamber at a (roughly) constant rate it is assumed that the pressure increased linearly with time. To measure the increase in pressure, $\mathrm{Xe}^{44+}$ charge transfer time measurements were repeated at the end of the experiment and compared with earlier results. Although the neutral density apparently changed by more than a factor of 5 over two weeks, the change in density during a particular measurement was small.

\section{F. Results and Discussion}

Table 5.1 summarizes the cross-section results for total (single + double) electron capture. Uncertainties for the thorium cross sections were estimated to be $10 \%$ uncertainty in fitting the data, $20 \%$ uncertainty in relative velocity, and $10 \%$ uncertainty in the neutral density. The uncertainties were added in quadrature to yield $25 \%$ total uncertainty. Uncertainties in the cross sections for xenon charge states (except $\mathrm{Xe}^{44+}$ ) are higher because the xenon measurements were made earlier; assuming a constant absolute increase in neutral density, the change in relative neutral density is higher earlier in the experiment when neutral density is lower. Uncertainty in the relative neutral density is estimated to be $30 \%$, yielding a total uncertainty of $37 \%$. Since the $\mathrm{Xe}^{44+}$ measurements were repeated at the end of the experiment (right after the $\mathrm{Ar}^{11+}$ 
calibration) the uncertainty for the $\mathrm{Xe}^{44+}$ cross section is assumed to be the same as for the thorium.

Table 5.1: Total electron capture data summary.

\begin{tabular}{|l|l|l|}
\hline Ion & $\begin{array}{l}\text { Total Electron Capture } \\
\text { Cross Section }\end{array}$ & Uncertainty \\
\hline \hline $\mathrm{Xe}^{35+}$ & $4.6 \times 10^{-14}$ & $1.7 \times 10^{-14}$ \\
\hline $\mathrm{Xe}^{43+}$ & $7.0 \times 10^{-14}$ & $2.6 \times 10^{-14}$ \\
\hline $\mathrm{Xe}^{44+}$ & $1.0 \times 10^{-13}$ & $2.5 \times 10^{-14}$ \\
\hline $\mathrm{Xe}^{45+}$ & $1.2 \times 10^{-13}$ & $3.6 \times 10^{-14}$ \\
\hline $\mathrm{Xe}^{46+}$ & $7.1 \times 10^{-14}$ & $2.6 \times 10^{-14}$ \\
\hline $\mathrm{Th}^{73+}$ & $1.8 \times 10^{-13}$ & $4.5 \times 10^{-14}$ \\
\hline $\mathrm{Th}^{74+}$ & $2.2 \times 10^{-13}$ & $5.5 \times 10^{-14}$ \\
\hline $\mathrm{Th}^{75+}$ & $2.2 \times 10^{-13}$ & $5.5 \times 10^{-14}$ \\
\hline $\mathrm{Th}^{76+}$ & $2.2 \times 10^{-13}$ & $5.5 \times 10^{-14}$ \\
\hline $\mathrm{Th}^{79+}$ & $1.9 \times 10^{-13}$ & $4.8 \times 10^{-14}$ \\
\hline
\end{tabular}

Figure 5.6 shows a plot of the data fit to $\sigma=\mathrm{Aq}^{\alpha}$. The uncertainty on the $\mathrm{Ar}^{11}$ was taken from the Kravis et al data [3]. The fit value of $\alpha$ was 1.2. Since the sources of error were mostly systematic rather than random, it is difficult to put an uncertainty on the value of $\alpha$. However, given the size of the error bars, this is consistent with a linear relation between charge and cross-section.

The fraction of double capture for thorium was obtained both by counting the number of electrons captured by an ion between sweeps when there was exactly one ion in the trap (using eq. 5.18) and by fitting the populations of the incident charge state and first charge exchange product state (using eq. 5.16 and 5.17). Double capture was found to average $25 \% \pm 9 \%$ of the total. Only the latter method could be used for analyzing the xenon and argon data, since there was virtually never exactly one xenon ion in the 


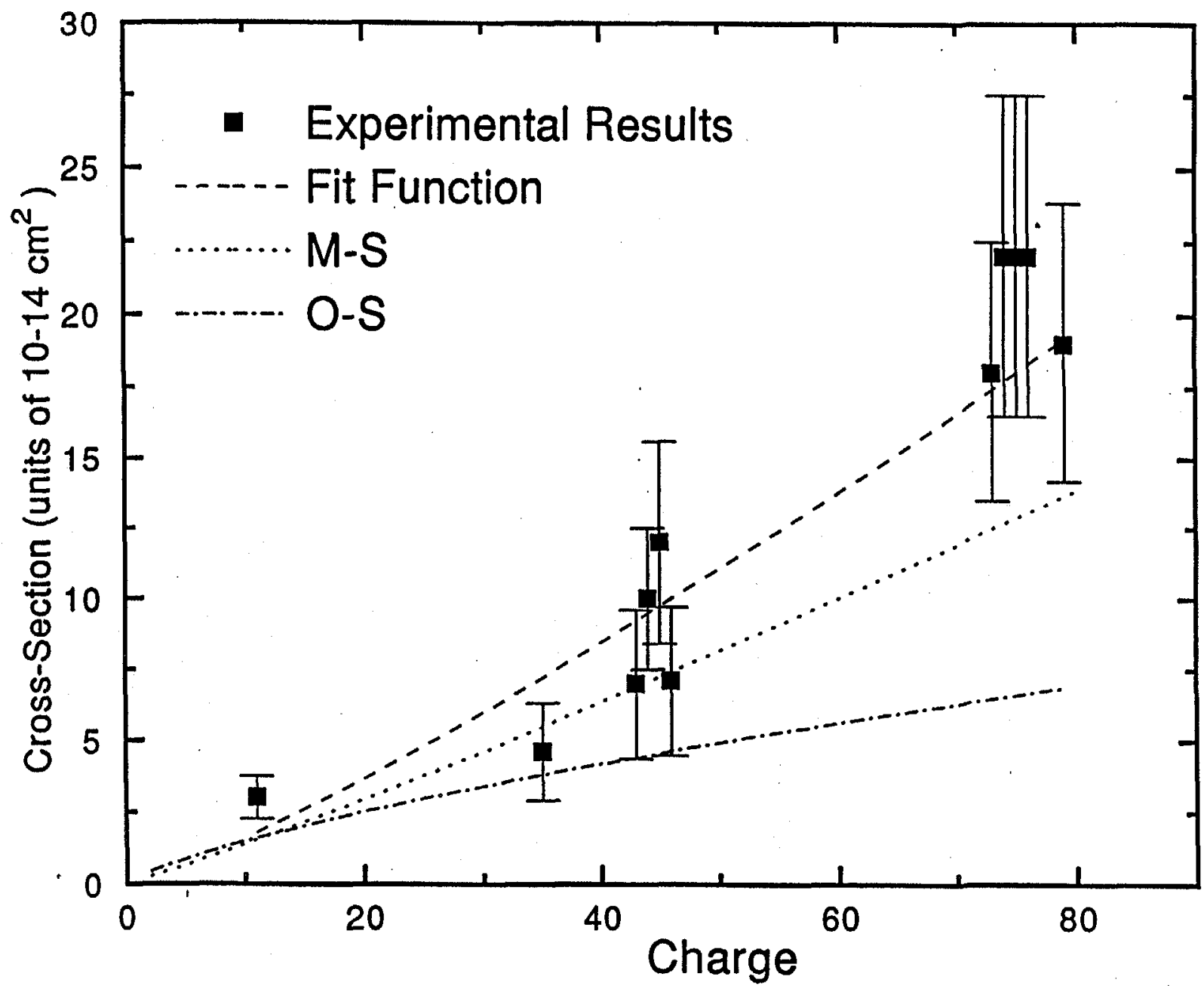

Figure 5.6: Total charge exchange cross-section data summary. The data is fitted to $\sigma=\mathrm{Aq}^{\alpha}$ (essentially the same fit function as used by Müller and Salzborn, except the ionization potential of the target is not a parameter because only one target gas was used). The fit value of $\alpha$ is 1.20 . The value and uncertainty for $\mathrm{Ar}^{11+}$ are taken from Kravis et. al. [3]. Also shown are the Müller and Salzborn (M-S) and Olson and Salop (O-S) predicted cross sections. 
trap, and the argon signals are too weak to see individual ions. Double capture was found to be $21 \% \pm 3 \%$ of the total for $\mathrm{Xe}^{35+}$ and average $23 \% \pm 6 \%$ of the total for $\mathrm{Xe}^{43+}$ to $\mathrm{Xe}^{46+}$. Double capture for $\mathrm{Ar}^{11+}$ was found to be $9 \% \pm 2 \%$ of the total, consistent with the Kravis data. Since the ratio of single to double capture is independent of neutral density, assuming the ratio is only weakly velocity dependent in the range studied, and that charge exchange cross sections and ratio of double capture to total capture are nearly the same for adjacent charge states, the uncertainties are purely statistical.

\section{G. Comparison with Theory}

As a general feature, measured cross-sections were higher than theoretical predictions for all the theories discussed.

The measured data for single electron capture and the Müller and Salzborn scaling law agree fairly well with respect to the dependence of charge capture crosssection on ion charge. Measured cross-sections are higher for all ion charge states. However, the scaling law is based on data taken at roughly 2 to 4 orders of magnitude higher energy than the current data, whereas the current data cross sections differ from the scaling law calculations by only about a factor of two. Even a very weak dependence of cross section on energy could explain this discrepancy.

The scaling law predicts that the fraction of double capture should monotonically decrease as a function of ion charge (single electron capture increases as $q^{1.17}$, double capture as $\mathrm{q}^{0.71}$ ). This appears not to be the case. A likely reason for this discrepancy is that Müller and Salzborn use the same scaling function regardless of target species (only the first ionization potential enters as a parameter), and much of their data is based on targets with many electrons. The scaling law predicts that triple electron capture increases as $\mathrm{q}^{2.1}$, much more rapidly than double capture. If electron capture occurs successively and the radius at which triple capture occurs increases faster than the radius at which double capture occurs in many electron atoms, the double electron capture will 
decrease (or at least increase more slowly). Clearly such a process cannot occur for two-electron atoms (or molecules) since triple electron capture is impossible.

The scaling law is the only model which makes a prediction as to the true double capture cross-section. The other models may predict an upper bound on the ratio of double to single capture, but they do not make any predictions with respect to the probability of autoionization.

The energy in this experiment is too high to expect the Langevin model to apply; however, it is worth noting that in Langevin-like collisions, no double electron capture is expected. Recall that in the Langevin model the motion of the neutral is dominated by the fourth order potential of the dipole induced on the neutral by the ion. After capturing an electron, there is a much stronger repulsive force between the heavy ion and the newly formed $\mathrm{H}_{2}{ }^{+}$ion. The $\mathrm{H}_{2}{ }^{+}$and the heavy ion will quickly separate, and no second electron transfer can occur. Similar considerations (based on conservation of energy) indicate that if electron capture occurs successively, for sufficiently low energies, no double capture is expected For example, if the first electron is captured from a hydrogen molecule to $\mathrm{Th}^{79+}$ ion at an internuclear separation of $3 \times 10^{-7} \mathrm{~cm}$ and the second is captured at $1 \times 10^{-7} \mathrm{~cm}$, there could be no double capture at interaction energies less than about $75 \mathrm{eV}$ (in the center of mass frame). This prediction does not apply if the two electrons are captured simultaneously, nor if the $\mathrm{H}_{2}{ }^{+}$dissociates into an $\mathrm{H}$ and an $\mathrm{H}^{+}$after a single electron is captured.

The data are consistent with the Grozdanov (classical) model with respect to the relation between cross-section and charge, although again the measured cross-sections are uniformly higher. This is unsurprising, as the Grozdanov cross-section was expected to be a lower bound to the true cross-section.

Classically, the cross-section for the initial capture of two electrons should simply increase as (q-1). However, without knowing what fraction of double capture is followed by autoionization vs. true double capture, it is impossible to compare this model directly with the experimental results. It is worth noting that if the hydrogen molecule dissociates into a hydrogen molecule and a proton after the first electron is 
captured, double capture should always result, because the electron of a hydrogen atom is less tightly bound (the ionization potential of atomic hydrogen is $13.6 \mathrm{eV}$ vs. $15.4 \mathrm{eV}$ for molecular hydrogen).

The final theory to be compared with experiment is the Olson and Salop theory. Recall the equation describing the critical radius for capture,

$R_{c}^{2} \exp \left[-2.648 \alpha R_{c} / z^{1 / 2}\right]=2.864 \times 10^{-4} z(z-1) v / f$

There is an ambiguity as to what value should be chosen for the Franck-Condon factor $f$. The value used here for calculating theoretical cross-sections is 0.1 , which is the value obtained from the theoretical curve in Olson and Salop's paper [9]. The experimental cross sections are significantly higher overall than the Olson-Salop cross sections. The experimental cross sections also show a stronger than predicted dependence of cross section on the ion charge. 


\section{CHAPTER VI}

\section{CONCLUSION}

Theories of charge exchange between highly charged ions and neutrals have been discussed.

The fast extraction of highly charged ions from EBIT and recapture into RETRAP have been described. The operation of the RETRAP system and the technique used to measure the populations of the individual ion charge states versus time have been described.

Data have been presented showing relative charge exchange rates for ions with initial charges ranging from $11+$ to $79+$ reacting with molecular hydrogen at energies of about $4 \mathrm{eV}$ in the center of mass frame. Ratios of single to double charge capture have been presented. Absolute charge capture cross sections have been calculated based on the published charge capture cross section of $\mathrm{Ar}^{11+}$.

After the conclusion of the experiments described herein the cylindrical trap was removed from the RETRAP chamber. The replacement trap has hyperbolic electrodes and ports for laser access. Although each type of trap has advantages over the other, the strongest reason for originally choosing a cylindrical trap was that the apertures for injecting ions can be made much larger. Analysis indicates that most ions are captured near the trap axis, so this advantage is considered to be less important. Operation of the new trap will be similar, the primary difference being that the new trap is expected to have less frequency spreading due to ion amplitude.

The extraction system for Super-EBIT will allow the possibility of experiments on ions of still higher charge states. A sympathetic cooling scheme in which highly charged ions are collisionally coupled with laser cooled $\mathrm{Be}^{+}$ions will allow experiments at much lower energies. Ion stacking will increase the number of ions which may be simultaneously contained in the trap. 
Future experiments at RETRAP will involve studies of hyperfine splittings in heavy hydrogenlike ions (such as has been measured for $\mathrm{Bi}^{82+}$ ) [27] and studies of ionion interactions in binary ionic systems ( $\mathrm{Be}^{+}$and highly charged ions) with a high value of the coulomb coupling constant. 


\section{REFERENCES}

1. D. A. Church, Phys. Rep. 228, 253 (1993).

2. N. Shimakura and K. Kimura, Phys. Rev. A 44, 1659 (1991).

3. S. Kravis, H. Saitoh, K. Okuno, K. Soejima, M. Kimura, I. Shimamura, Y. Okuno, K. Soejima, M. Kimura, I. Shimamura, Y. Awaya, Y. Kaneko, M. Oura, and N. Shimakura, Phys. Rev. A 52, 1206 (1995).

4. A. Wolf, J. Berger, M. Bock, D. Habs, B. Hochadel, G. Klingus, G. Neureither, U. Schramm, D. Schwalm, E. Szmola, A. Muller, MM. Wagner and R. Schuch, Z. Phys. D. 21, S69 (1991).

5. B. Hochadel, F. Albrecht, M. Grieser, D. Habs, D. Schwalm, E. Szmola, and A. Wolf, Nucl. Instrum. Methods Phys. Res. A 343, 401 (1994).

6. E.S. Maramand, J.L. Terry, Atomic Processes in Plasmas, AIP conference proceedings 257 (AIP, New York, 1992).

7. M. A. Levine, R.E. Marrs, and R. W. Schmieder, Nucl. Instrum. Methods Phys. Res. A 237, 429 (1985).

8. T. P. Grozdanov, J Phys. B 13, 3835 (1980).

9. R. E. Olson and A. Salop, Phys. Rev. A 14, 579 (1976).

10. G. Gioumousis and D. P. Stevenson, J. Chem. Phys. 29, 294 (1958).

11. A Müller and E. Salzborn Phys. Lett. 62A, 391 (1977)

12. R. K. Janev and L. P. Presnyakov, Phys. Reports 70, 1 (1981).

13. H. Cederquist, H. Andersson, G. Astner, P. Hvelplund, and J. O. P. Pedersen, Phys. Rev. Lett. 62, 1465 (1989).

14. M. Barat and P. Roncin, J. Phys. B 25, 2205 (1982).

15. M. A. Levine, R. E. Marrs, J.R. Henderson, D.A. Knapp and M. B. Schneider, Phys. Scr. T22, 157 (1988).

16. I. G. Brown, J.E. Galvin, R.A. McGill, and R.T. Wright, Appl. Phys. Lett. 49, 16 (1968). 
17. S. R. Elliot and R. E. Marrs, Nucl. Instrum. Methods Phys. Res. B 100, 529 (1995).

18. R. Marrs, Personal communication

19. P. Dahl, Introduction to Electron and Ion Optics, (Academic Press, New York 1973).

20. L. Brown and G. Gabrielse, Rev. Mod. Phys. 58, 233 (1986).

21. G. Gabrielse, L. Haarsma and S. L. Rolston, Int. J. Mass Spectrom. Ion Process 88, 319 (1989).

22. A. Lee, Rev. Sci. Instrum. 60, 3315 (1989).

23. J. Steiger, G. Weinberg, B. Beck, D.A. Church, J. McDonald and D. Schneider, Nucl. Instrum. Methods Phys. Res. B 98, 569 (1995).

24. S. Ichimaru and M. N. Rosenbluth, Phys. Fluids 13, 2278 (1970).

25. B. R. Beck, J. Fajans and J. H. Malmberg, Phys. Rev. Lett. 68, 317 (1992).

26. S. Tu and D. A. Church, Chem. Phys. Lett. 174, 301 (1990).

27. I. Klaft, S. Borneis, T. Engel, B. Fricke, R. Grieser, G. Huber, T. Kühl, D. Marx, R. Neumann, S. Scröder, P. Seelig, and L. Völker, Phys. Rev. Lett. 73, 2425 (1994). 


\section{VITA}

\section{George Morton Weinberg}

the second child of Morton and Joni Weinberg. He grew up in Maryland and graduated from Bethesda Chevy Chase High School in May 1983.

He attended the University of Illinois at Urbana-Champaign from 1983 to 1987 and graduated with a B.A. in physics.

He became a physics graduate student at Texas A\&M in the fall of 1987 . Since July 1992 he has been a student guest at the Lawrence Livermore National Lab in Livermore, California, where he conducted the research described in this dissertation. Mail may be sent to him care of Joni Weinberg at 709 Elm Street, Washington II 61571. 
